\title{
Odd sphere bundles, symplectic manifolds, and their intersection theory
}

\author{
Hiro Lee Tanaka and Li-Sheng Tseng
}

\begin{abstract}
We introduce and investigate a new perspective which relates invariants of a symplectic manifold to topological invariants of certain odd-dimensional sphere bundles over the symplectic manifold. Specifically, we show that the novel symplectic $A_{\infty}$-algebras of differential forms recently found by Tsai-Tseng-Yau are in fact equivalent to the standard de Rham differential graded algebra of the odd sphere bundles when the cohomology class of the symplectic form is integral. As applications of this equivalence, we deduce for a closed symplectic manifold that Tsai-Tseng-Yau's symplectic $A_{\infty}$-algebras satisfy the Calabi-Yau property and argue that they can be used to define an intersection theory for cycles built from coisotropic/isotropic submanifolds. We further demonstrate that these symplectic $A_{\infty}$-algebras satisfy several functorial properties and lay the groundwork for addressing Weinstein functoriality.
\end{abstract}

1 Introduction $\quad 214$

$\begin{array}{llr}2 & \text { Recollections } & \mathbf{2 1 9}\end{array}$

$\begin{array}{lll}2.1 & \text { Form decomposition } & 219\end{array}$

2.2 The cochain complex $\mathcal{F}_{p}^{\bullet} \quad 221$

2.3 The $A_{\infty}$-algebra structure 223

3 Equivalence of algebras $\quad 224$

$\begin{array}{lll}3.1 & \text { Definition of } \mathcal{C} & 224\end{array}$

$\begin{array}{lll}3.2 & \mathcal{C} \text { is equivalent to } \mathcal{F} & 226\end{array}$

3.3 Proof of Theorem $3.8 \quad 232$

4 Calabi-Yau structure on $\mathcal{F} \quad 237$

5 Homology and intersection when $\omega$ is integral 244

$\begin{array}{lll}5.1 & \text { Recollections on currents } & 245\end{array}$ 
5.2 Homology of primitive currents

5.3 Intersection theory via lifts to $E$

5.4 Example: Kodaira-Thurston four-fold.

6 Functoriality of $\mathcal{F}$

6.1 On objects

6.2 On morphisms

6.3 On orthogonal homotopies

6.4 Sheaf property

6.5 As sheaf cohomology

6.6 Looking forward: Weinstein functoriality

A Proof that $\operatorname{Cone}(\omega)$ is equivalent to differential forms on a sphere bundle

References

\section{Introduction}

Recently, Tsai-Tseng-Yau [16] introduced a family of algebras of differential forms for any symplectic manifold $M$. These algebras - denoted $\mathcal{F}_{p}(M)$, with parameter $p$ taking integral values from zero to $\operatorname{dim} M / 2$ - have several notable properties. First, each $\mathcal{F}_{p}(M)$ is an $A_{\infty}$-algebra. Second, the differential of the algebra consists of both first- and second-order differential operators, while the product interestingly involves derivatives. Finally, their cohomologies, $F^{p} H^{*}(M)$, are all finite-dimensional-they were named the $p$-filtered cohomologies of $M$ for short [16]. These filtered cohomology rings can vary with the symplectic structure $\omega[18,16]$. The filtered cohomologies also have physical applications. In fact, they arise naturally when considering mirror symmetry of non-Kähler manifolds; in particular, they appear in the deformation theory of type IIA supersymmetric string solutions that are non-Kähler symplectic $[19,14]$.

In this paper, we equate the algebras $\mathcal{F}_{p}$ (in the $A_{\infty}$-algebra sense) to a standard de Rham differential graded algebra - not on the original symplectic manifold $M$, but on certain odd-dimensional sphere bundles over $M$. This equivalence turns out to give a simple, topological description of $\mathcal{F}_{p}$, 
and makes manifest several useful properties of $\mathcal{F}_{p}$ (including some functorial properties).

We can motivate the existence of such an alternative description for $\mathcal{F}_{p}$ on $\left(M^{2 n}, \omega\right)$ from a broader point of view. For a symplectic manifold $M$, the symplectic structure and its powers constitute a distinguished set of forms:

$$
\left\{\omega, \omega^{2}, \ldots, \omega^{n}\right\} \in \Omega^{*}(M) .
$$

Special forms also arise in geometry as representatives of characteristic classes of bundles over $M$. Assuming $\omega$ is also in an integral cohomology class, it is then natural to combine these two types of forms by considering bundles over $M$ with characteristic classes given by powers of $\omega$.

Explicitly, for the distinguished two-form $\omega$, consider the line bundle $L$ over $M$ with $c_{1}=(1 / 2 \pi) \omega$. This $L$ is the well-known prequantum line bundle in geometric quantization, and has an associated circle bundle with Euler class $e=\omega$. More generally, for the 2(p+1)-form $\omega^{p+1}$, we have the associated direct sum vector bundle $L^{\oplus p+1}$, and we will focus in particular on the associated closed $S^{2 p+1}$ bundle over $M$ :

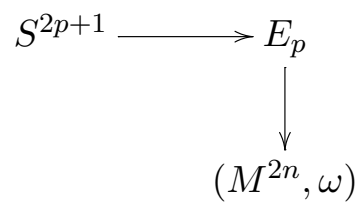

with its Euler class given by $\omega^{p+1}$. Given that $E_{p}$ is built from $M$ and the symplectic form $\omega$, and nothing more, one can ask how the topology of $E_{p}$ relates to the symplectic geometry of $M^{2 n}$ :

$$
\text { Topological data of } E_{p}<-? \text { Symplectic data of }\left(M^{2 n}, \omega\right)
$$

Specifically,

(1) What symplectic data are encoded in the topology of the odd sphere bundle $E_{p}$ ?

(2) Can we use the topology of these sphere bundles to gain new insights into the known symplectic invariants on $\left(M^{2 n}, \omega\right)$ ?

In this paper, we begin to address such questions by analyzing the cohomology and the differential topology of $E_{p}$. First, the real cohomology of the 
sphere bundle - equivalently, its de Rham cohomology - is well-known to fit into the Gysin sequence:

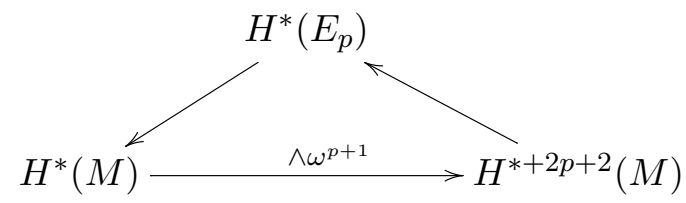

where again $\omega^{p+1}$ is the Euler class of $E_{p}$. This exact triangle identifies $H^{*}\left(E_{p}\right)$ non-canonically as the direct sum of kernels and cokernels of the $\omega^{p+1}$ map between the de Rham cohomologies of $M$. Strikingly, exactly the same triangle diagram also appeared in [16] for the symplectic $p$-filtered cohomologies of differential forms, $F^{p} H^{*}(M)$; so we can conclude from (2) that

$$
\begin{aligned}
H^{*}\left(E_{p}\right) & \cong \operatorname{coker}\left[\omega^{p+1}: H^{*}(M) \rightarrow H^{*}(M)\right] \oplus \operatorname{ker}\left[\omega^{p+1}: H^{*}(M) \rightarrow H^{*}(M)\right] \\
& \cong F^{p} H^{*}(M)
\end{aligned}
$$

Beyond the cohomology of $E_{p}$, we can also consider the de Rham algebra of $E_{p}$. When $[\omega]$ is integral, a standard application of the Čech-de Rham double complex shows that

$$
\Omega^{\bullet}\left(E_{p}\right) \simeq \operatorname{Cone}\left(\omega^{p+1}\right)
$$

where Cone $\left(\omega^{p+1}\right)$ is the mapping cone of the map between de Rham chain complexes:

$$
\wedge \omega^{p+1}: \Omega^{\bullet}(M)[-2 p-2] \rightarrow \Omega^{\bullet}(M) .
$$

We refer the interested reader to the proof in the Appendix.

The cone algebra Cone $\left(\omega^{p+1}\right)$ can be endowed with the structure of a commutative differential graded algebra (cdga). It is also well-defined for any $\omega$ whether integral or not. At first glance, this cone cdga is very different from the $A_{\infty}$-algebra $\mathcal{F}_{p}$, but both fit into the same long exact sequence of cohomology groups (hence have isomorphic cohomology). This raises the question whether $\mathcal{F}_{p}$ and Cone $\left(\omega^{p+1}\right)$ represent two distinct symplectic algebras on $(M, \omega)$. We prove that they are in fact equivalent:

Theorem 1.1. For each $p$, there exists a natural equivalence of $A_{\infty}$-algebras

$$
\mathcal{F}_{p} \simeq \operatorname{Cone}\left(\omega^{p+1}\right) .
$$


Here, naturality is with respect to smooth immersions $f: M \rightarrow M^{\prime}$ such that $\operatorname{dim} M=\operatorname{dim} M^{\prime}$ and $f^{*} \omega^{\prime}=\omega$.

Moreover, this equivalence suggests that $\mathcal{F}_{p}$ should come with a nondegenerate pairing when $M$ is compact - after all, when [ $\omega]$ is integral, $\Omega\left(E_{p}\right)$ has a non-degenerate pairing by Poincaré Duality. To that end, we prove:

Theorem 1.2. Each $A_{\infty}$-algebra $\mathcal{F}_{p}$ is Calabi-Yau whenever $M$ is compact.

In other words, each $\mathcal{F}_{p}$ admits a cyclically invariant pairing, and this theorem holds for all symplectic structures on $M$.

Remark 1.3. In introducing the sphere bundles, we needed to assume $[\omega]$ is integral. However, the above theorems hold for any $\omega$. In particular, the content of Theorem 1.2 is twofold: We exhibit the Calabi-Yau property in the absence of a sphere bundle, and we also write an explicit Calabi-Yau structure making the dependence on $\omega$ transparent. As a bonus, when $[\omega]$ is integral, we verify that the motivating Poincaré duality pairing on $E$ is compatible with the pairing we construct (via the equivalence of Theorem 1.1).

At this point, two natural questions arise from these theorems.

First: Is there an intersection theory associated to each $\mathcal{F}_{p}$ ? After all, common examples of Calabi-Yau algebras are differential forms on compact, oriented manifolds, where the Calabi-Yau pairing encodes the intersection theory of oriented submanifolds. Since the sequence of Calabi-Yau algebras $\mathcal{F}_{p}$ depends on the choice of $\omega$, an answer to our question amounts to looking for an intersection theory tailored to symplectic manifolds - and one not involving holomorphic curve theory.

In Section 5, we begin to interpret the intersection pairing when $\omega$ is an integral cohomology class and $p=0$. The interpretation is as follows: Consider the contact circle bundle $E$ associated to the line bundle classified by $\omega$. Given an isotropic $I$ inside $M$, one can lift $I$ to a section $\tilde{I}$ of $E$ because $\left.\omega\right|_{I}=0$. As it turns out, isotropics naturally pair with coisotropics $C \subset M$, but $C$ may have some non-trivial, symplectic boundary. (This is a manifestation of the fact that the Poincaré dual form of $C$ may not be closed under the de Rham differential.) Regardless, our work suggests that $C$ lifts to a submanifold $\tilde{C} \subset E$ in the circle bundle which no longer has boundary. Then the intersection of $\tilde{I}$ with $\tilde{C}$ - given by the usual intersection pairing on $E$-is the Calabi-Yau pairing on $\mathcal{F}_{0}$.

Remark 1.4. While the above theorems imply that the symplectic $A_{\infty^{-}}$ algebras $\mathcal{F}_{p}$ are only invariants of the cohomology class of $\omega$, and not $\omega$ itself (see Corollary 6.2), the geometric construction of the intersection pairing 
involves knowledge of $\omega$ itself. In particular, this construction suggests that one should further contemplate whether the differential geometry (rather than the topology) of the odd sphere bundle induced by $\omega$ encodes symplectic data of $M$. As an example, in the $p=0$ case, the circle bundle has a contact structure, $\theta$, such that $\omega=d \theta$. Hence, one can study this contact geometry in relation to the symplectic geometry of the base. And as shown by Bourgeois [2], the contact homology of the circle bundle $E_{p=0}$ relates directly to the genus zero Gromov-Witten invariants on $(M, \omega)$.

Second: What formal properties do the $\mathcal{F}_{p}$ satisfy? For instance, what kinds of symplectic morphisms induce maps between them?

We prove that the algebras $\mathcal{F}_{p}$ enjoy several formal properties in Section 6. For instance, since there are natural quotient maps Cone $\left(\omega^{p+1}\right) \rightarrow$ Cone $\left(\omega^{p}\right)$, one obtains a sequence of $A_{\infty}$-algebra maps that go in a reverse direction to the filtration that defines $\mathcal{F}_{p}$ :

$$
\ldots \rightarrow \mathcal{F}_{p} \rightarrow \mathcal{F}_{p-1} \rightarrow \ldots \rightarrow \mathcal{F}_{0} .
$$

Using the equivalence of Theorem 1.1 to render $\mathcal{F}_{p}$ a commutative (and not just $A_{\infty}$ ) algebra, this sequence of algebra maps suggests that the $\mathcal{F}_{p}$ can be given a simple interpretation in terms of derived geometry - they encode higher order neighborhoods around the subvariety of $\operatorname{Spec}(\Omega(M))$ determined by $\omega$.

Furthermore, we begin to develop the functorial properties of the assignment $M \mapsto \operatorname{Cone}\left(\omega^{\bullet}\right)$. For instance, this assignment is functorial with respect to maps $f: M \rightarrow M^{\prime}$ such that $f^{*} \omega=\omega^{\prime}$. Moreover, the assignment $M \mapsto \mathcal{F}_{p}(M)$ forms a sheaf of cdgas on the site of symplectic manifolds with smooth, open embeddings that are symplectomorphisms onto their images. Finally, we begin to see the seeds of Weinstein functoriality: For any Lagrangian submanifold $L \subset\left(M_{1} \times M_{2},-\omega_{1} \oplus \omega_{2}\right)$, one can define a cdga Cone $_{L}\left(\omega^{p+1}\right)$ receiving an algebra map from each of the Cone $M_{i}\left(\omega^{p+1}\right)$-in particular, each Lagrangian correspondence defines a bimodule for the algebras $\mathcal{F}_{p}\left(M_{i}\right)$. It will be a subject of later work to show that this assignment sends Lagrangian correspondences to tensor products of bimodules.

Acknowledgments. We thank Kevin Costello, Rune Haugseng, Nitu Kitchloo, Si Li, Richard Schoen, Xiang Tang, Chung-Jun Tsai, and ShingTung Yau for helpful discussions. We also would like to thank the journal's referee for providing numerous suggestions for improving the original manuscript. This research is supported in part by National Science Foundation under Award No. DMS-1400761 of the first author and a Simons Collaboration Grant of the second author. We are also grateful for the hospitality 
of the Perimeter Institute for Theoretical Physics and Harvard University's Center for Mathematical Sciences and Applications while this research took place. Research at Perimeter Institute is supported by the Government of Canada through the Department of Innovation, Science and Economic Development and by the Province of Ontario through the Ministry of Research and Innovation.

\section{Recollections}

Here we recall the constructions and results from [17], [18], [16]. This will allow us to set some notation, and to recall the $A_{\infty}$-operations for the algebras $\mathcal{F}_{p}$. (Here, $p$ can be any integer between 0 and $n$, inclusive.)

Notation: We will often denote a cochain complex simply by a letter such as $\Omega$, referring to its degree $k$ part by $\Omega^{k}$. To emphasize that a cochain complex is graded, we will also write $\Omega^{\bullet}$ instead of $\Omega$. Finally, when a cochain complex such as $\mathcal{F}_{p}$ or $\Omega$ depends on a choice of manifold $M$, we may write $\Omega(M)$ to indicate the dependence on $M$; for brevity and space, we will usually make the dependence on $M$ implicit and simply write $\Omega$.

\subsection{Form decomposition}

Fix a $2 n$-dimensional symplectic manifold $\left(M^{2 n}, \omega\right)$. Since $\omega$ defines an isomorphism between 1-forms and vector fields, $\omega$ itself is identified with a bivector field (the Poisson bivector field), under this isomorphism. There are three basic operations on de Rham forms on $M$ :

(1) $L: \Omega^{k} \rightarrow \Omega^{k+2}$ sends $\eta \mapsto \omega \wedge \eta$.

(2) $\Lambda: \Omega^{k} \rightarrow \Omega^{k-2}$ sends a $k$-form $\eta$ to the interior product with the Poisson bivector field.

(3) $H: \Omega^{k} \rightarrow \Omega^{k}$ sends a $k$-form $\eta$ to $(n-k) \eta$.

Together, $\{L, \Lambda, H\}$ generate an $s l_{2}$ Lie algebra acting on forms. The highest weight forms under this action are called primitive forms, whose space we shall denote by $P^{\bullet}$. If $\beta_{k} \in P^{k}$ is a primitive $k$-form, then its interior product with the Poisson bivector field vanishes, i.e. $\Lambda \beta_{k}=0$. As usual with $s l_{2}$ representations, any $k$-form $\eta_{k} \in \Omega^{k}$ can be expressed as a sum of primitive forms wedged with powers of $\omega$ :

$$
\eta_{k}=\sum_{k=2 j+s} L^{j} \beta_{s}=\beta_{k}+L \beta_{k-2}+\ldots+L^{p} \beta_{k-2 p}+\ldots
$$


This Lefschetz decomposition of $\Omega^{\bullet}$ can be arranged in a "pyramid" diagram as shown in Figure 1. (This can be compared to the well-known $(p, q)$ "diamond" of forms on complex manifolds.) Associated with this Lefschetz decomposition, we define four more operations:

(4) $L^{-p}$ : the negative power of $L$ with $p>0$. Roughly, it is the "inverse" of $L^{p}$ and removes $\omega^{p}$ from each term of the decomposition. Explicitly,

$$
L^{-p} \eta_{k}=\sum_{k=2 j+s} L^{-p} L^{j} \beta_{s}=\beta_{k-2 p}+L \beta_{k-2 p-2}+\ldots
$$

(5) $*_{r}$ : the natural reflection action about the central axis of the pyramid diagram in Figure 1. The operation is explicitly defined on each term of the Lefschetz decomposition by

$$
*_{r}\left(L^{j} \beta_{s}\right)=L^{n-j-s} \beta_{s} .
$$

It is worthwhile to note that in terms of $*_{r}$,

$$
L^{-p}=*_{r} L^{p} *_{r}
$$

that is, $L^{-p}$ is the $*_{r}$ conjugate of $L^{p}$. Also, acting on $\Omega$, we have $*_{r} \Omega^{k}=\Omega^{2 n-k}$ as expected.

(6) $\Pi^{p}$ : a projection operator that keeps only terms up to the $p$ th power of $\omega$ in the Lefschetz decomposition:

$$
\Pi^{p} \eta_{k}=\sum_{j=0}^{p} L^{j} \beta_{s}=\beta_{k}+\ldots+L^{p} \beta_{k-2 p}
$$

Alternatively, $\Pi^{p}$ can be defined as

$$
\Pi^{p}=1-L^{p+1} \circ L^{-(p+1)} .
$$

(7) $\Pi^{p *}$ : the $*_{r}$ conjugate of $\Pi^{p}$ defined by

$$
\Pi^{p *}=*_{r} \Pi^{p} *_{r}=1-L^{-(p+1)} \circ L^{p+1} .
$$

The decomposition by powers of $\omega$ allows us to group together forms that contain terms only up to certain powers of $\omega$.

Definition 2.1. Define the space of $p$-filtered $k$-forms to be

$$
F^{p} \Omega^{k}:=\Pi^{p} \Omega^{k}
$$




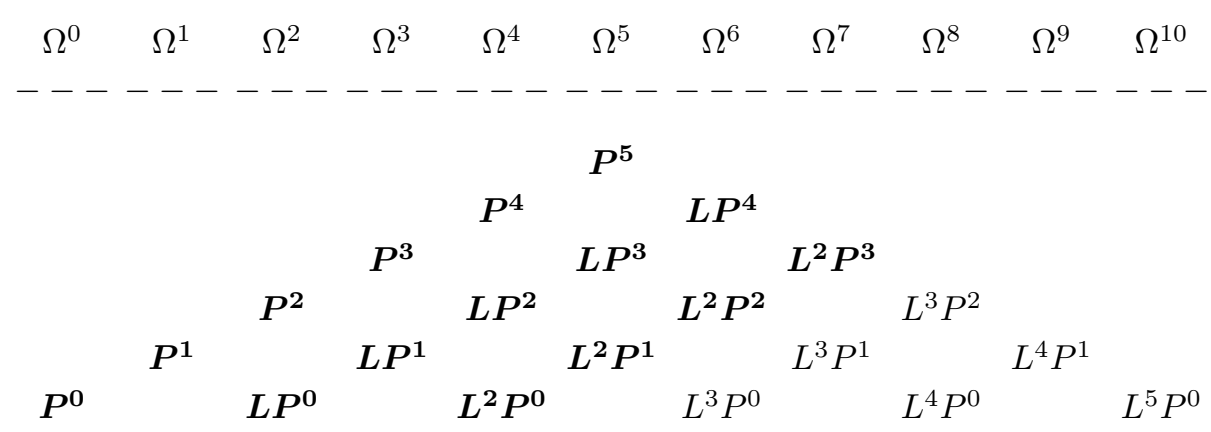

Figure 1: The decomposition of forms into a "pyramid" diagram in dimension $2 n=10$. Note that there is a natural reflection symmetry action about the $\Omega^{n=5}$ column. This is the $*_{r}$ action defined in (5). To illustrate filtered forms, we have bold-faced those elements in $F^{p} \Omega$ for $p=2$ here.

for $p \in\{0,1, \ldots, n\}$.

The filtered $F^{p} \Omega^{k}$ spaces give us a natural filtration of $\Omega^{k}$ :

$$
P^{k}:=F^{0} \Omega^{k} \subset F^{1} \Omega^{k} \subset F^{2} \Omega^{k} \subset \ldots \subset F^{n} \Omega^{k}=\Omega^{k} .
$$

Note that the 0 -filtered forms are precisely primitive forms, and that $F^{n} \Omega^{k}=$ $\Omega^{k}$.

Lemma 2.2. The following are equivalent conditions for $p$-filtered forms:

(i) $\alpha_{k} \in F^{p} \Omega^{k}$;

(ii) $\Lambda^{p+1} \alpha_{k}=0$ (or equivalently, $L^{-(p+1)} \alpha_{k}=0$ );

(iii) $L^{n+p+1-k} \alpha_{k}=0$;

(iv) $L^{p+1} *_{r} \alpha_{k}=0$.

Remark 2.3. The $F^{p} \Omega^{k}$ space is only non-zero for $k=0,1, \ldots,(n+p)$. See Figure 1.

\subsection{The cochain complex $\mathcal{F}_{p}^{\bullet}$}

There are natural differential operators $\left(d_{+}, d_{-}\right)$that map between $p$-filtered forms. We recall the key ingredients here:

1. On symplectic manifolds, the exterior differential has a natural decomposition

$$
d=\partial_{+}+\omega \wedge \partial_{-} .
$$


where $\partial_{+}: L^{j} P^{s} \rightarrow L^{j} P^{s+1}$ and $\partial_{-}: L^{j} P^{s} \rightarrow L^{j} P^{s-1}$. Note in particular when $j=0, \partial_{+}$and $\partial_{-}$map primitive forms into primitive forms. The pair of operators $\left(\partial_{+}, \partial_{-}\right)$are analogous to the Dolbeault operators on complex manifolds. They square to zero $\left(\partial_{+}^{2}=\partial_{-}^{2}=0\right)$, commute with $L\left(\left[L, \partial_{+}\right]=\left[L, L \partial_{-}\right]=0\right)$, and also graded commute after applying $L: L \partial_{+} \partial_{-}=-L \partial_{-} \partial_{+}$.

2. On $p$-filtered forms, we can define two natural linear operators:

$$
\begin{aligned}
& d_{+}=\Pi^{p} \circ d, \\
& d_{-}=*_{r} d *_{r},
\end{aligned}
$$

such that $d_{+}: F^{p} \Omega^{k} \rightarrow F^{p} \Omega^{k+1}$ and $d_{-}: F^{p} \Omega^{k} \rightarrow F^{p} \Omega^{k-1}$. Both operators square to zero $\left(\left(d_{+}\right)^{2}=\left(d_{-}\right)^{2}=0\right)$. In fact, when acting on primitive forms, $d_{ \pm} P^{k}=\partial_{ \pm} P^{k}$. Furthermore, $\left(d_{+}, d_{-}\right)$is related to $\left(\partial_{+}, \partial_{-}\right)$by

$$
\begin{aligned}
d_{-} & =\partial_{-}+\partial_{+} L^{-1} \\
\left(\partial_{+} \partial_{-}\right) d_{+} & =d_{-}\left(\partial_{+} \partial_{-}\right)=0
\end{aligned}
$$

The differentials $\left(d_{+}, d_{-}, \partial_{+} \partial_{-}\right)$can be used to write down an elliptic complex for $F^{p} \Omega^{\bullet}$ :

Theorem 2.4. [Section 3 of [16]] The differential complex

$$
\begin{aligned}
0 \rightarrow F^{p} \Omega^{0} \stackrel{d_{+}}{\longrightarrow} F^{p} \Omega^{1} & \stackrel{d_{+}}{\longrightarrow} \ldots \stackrel{d_{+}}{\longrightarrow} F^{p} \Omega^{n+p} \stackrel{\partial_{+} \partial_{-}}{\longrightarrow} \\
& \stackrel{\partial_{+} \partial_{-}}{\longrightarrow} F^{p} \Omega^{n+p} \stackrel{d_{-}}{\longrightarrow} F^{p} \Omega^{n+p-1} \stackrel{d_{-}}{\longrightarrow} \ldots \stackrel{d_{-}}{\longrightarrow} F^{p} \Omega^{0} \rightarrow 0
\end{aligned}
$$

is elliptic for all $p \in\{0,1, \ldots, n\}$.

As a corollary of the ellipticity of the complex, the associated filtered cohomologies labelled by

$$
F^{p} H=\left\{F^{p} H_{+}^{0}, \ldots, F^{p} H_{+}^{n+p}, F^{p} H_{-}^{n+p}, \ldots, F^{p} H_{-}^{0}\right\}
$$

are all finite-dimensional on a closed symplectic manifold and also have Hodge theory associated to them.

The cochain complex $\mathcal{F}_{p}$ is defined following the elliptic complex:

(9) $\mathcal{F}_{p}=\left(F^{p} \Omega^{0} \stackrel{d_{+}}{\longrightarrow} \ldots \stackrel{d_{+}}{\longrightarrow} F^{p} \Omega^{n+p} \stackrel{-\partial_{+} \partial_{-}}{\longrightarrow}\right.$

$$
\left.\stackrel{-\partial_{+} \partial_{-}}{\longrightarrow} \overline{F^{p} \Omega^{n+p}} \stackrel{-d_{-}}{\longrightarrow} \overline{F^{p} \Omega^{n+p-1}} \stackrel{-d_{-}}{\longrightarrow} \ldots \stackrel{-d_{-}}{\longrightarrow} \overline{F^{p} \Omega^{0}}\right) .
$$


As vector spaces $\overline{F^{p} \Omega^{k}}=F^{p} \Omega^{k}$. The extra bar is only inserted to distinguish the second set of $F^{p} \Omega^{\bullet}$ in the complex from the first. Also, the additional minus signs in some of the differentials are needed to satisfy the $A_{\infty}$ relations described below. At times, we will denote $\mathcal{F}_{p}^{\bullet}$ simply as $\mathcal{F}^{\bullet}$, with

$$
\mathcal{F}^{j}= \begin{cases}\frac{F^{p} \Omega^{j}}{F^{p} \Omega^{2 n+2 p+1-j}} & j \leq n \\ 0 & j \geq n+p+1 \\ 0 & j<0, j>2 n+2 p+1 .\end{cases}
$$

The notation here is that $\overline{F^{p} \Omega^{k}}$ has degree $2 n+2 p+1-k$ in this complex, while $F^{p} \Omega^{k}$ has degree $k$. If ambiguity may arise whether a $p$-filtered $k$-form is an element of $\mathcal{F}^{k}$ or $\mathcal{F}^{2 n+2 p+1-k}$, we will add a bar and write $\bar{\alpha}_{k} \in \overline{F^{p} \Omega^{k}}$ to denote an element in $\mathcal{F}^{2 n+2 p+1-k}$.

\subsection{The $A_{\infty}$-algebra structure}

We use the sign convention that a series of operations

$$
m^{l}: \mathcal{F}^{\otimes l} \rightarrow \mathcal{F}[2-l]
$$

satisfies the $A_{\infty}$ relations if and only if

$$
\sum(-1)^{r+s t} m^{r+t+1}\left(\mathrm{id}^{\otimes r} \otimes m^{s} \otimes \mathrm{id}^{\otimes t}\right)=0 .
$$

As an example, if $m^{l}=0$ for $l \geq 3$, then the data $\left(\mathcal{F},\left(m^{l}\right)\right)$ define an ordinary differential graded algebra (dga).

\section{The $m^{2}$ operation.}

1. For $a_{1} \in F^{p} \Omega^{k_{1}}=\mathcal{F}_{p}^{k_{1}}, a_{2} \in F^{p} \Omega^{k_{2}}=\mathcal{F}_{p}^{k_{2}}$, and $k_{1}+k_{2} \leq n+p$, we set

$$
m^{2}\left(a_{1}, a_{2}\right)=\Pi^{p}\left(a_{1} \wedge a_{2}\right)
$$

where $\Pi^{p}$ is the projection to the $p$-filtered components defined above.

2. For $a_{1} \in F^{p} \Omega^{k_{1}}=\mathcal{F}_{p}^{k_{1}}, a_{2} \in F^{p} \Omega^{k_{2}}=\mathcal{F}_{p}^{k_{2}}$, and $k_{1}+k_{2}>n+p$, define

$$
\begin{aligned}
D_{L}\left(a_{1}, a_{2}\right)=-d & L^{-(p+1)}\left(a_{1} \wedge a_{2}\right) \\
& +\left(L^{-(p+1)} d a_{1}\right) \wedge a_{2}+(-1)^{j} a_{1} \wedge\left(L^{-(p+1)} d a_{2}\right) .
\end{aligned}
$$

Then,

$$
m^{2}\left(a_{1}, a_{2}\right)=\Pi^{p} *_{r}\left(D_{L}\left(a_{1}, a_{2}\right)\right) .
$$


In other words, the $m^{2}$ operation measures the difference between the action of $d L^{-(p+1)}$ and the derivation of $L^{-(p+1)} d$ on the wedge product of filtered forms. (It then picks out the $p$-filtered part of the $*_{r}$ reflection.)

3. For $a_{1} \in F^{p} \Omega^{k_{1}}=\mathcal{F}_{p}^{k_{1}}$ and $a_{2} \in \overline{F^{p} \Omega^{k_{2}}}=\mathcal{F}_{p}^{2 n+2 p+1-k_{2}}$, we set

$$
m^{2}\left(a_{1}, a_{2}\right)=(-1)^{k_{1}} *_{r}\left(a_{1} \wedge\left(*_{r} a_{2}\right)\right) .
$$

4. For $a_{1} \in \overline{F^{p} \Omega^{k_{1}}}=\mathcal{F}_{p}^{2 n+2 p+1-k_{1}}$ and $a_{2} \in F^{p} \Omega^{k_{2}}=\mathcal{F}_{p}^{k_{2}}$, we set

$$
m^{2}\left(a_{1}, a_{2}\right)=*_{r}\left(\left(*_{r} a_{1}\right) \wedge a_{2}\right) .
$$

This implies that $m^{2}$ is graded commutative:

$$
m^{2}\left(a_{1}, a_{2}\right)=(-1)^{a_{1} a_{2}} m^{2}\left(a_{2}, a_{1}\right) .
$$

5. And for degree reasons, if $a_{1} \in \overline{F^{p} \Omega^{k_{1}}}$ and $a_{2} \in \overline{F^{p} \Omega^{k_{2}}}$, then

$$
m^{2}\left(a_{1}, a_{2}\right)=0 \text {. }
$$

The $m^{3}$ operation. Let $a_{i} \in \mathcal{F}_{p}^{k_{i}}$. We set

$m^{3}\left(a_{1}, a_{2}, a_{3}\right)=\left\{\begin{array}{cc}\Pi^{p} *_{r}\left[a_{1} \wedge L^{-(p+1)}\left(a_{2} \wedge a_{3}\right)\right. & k_{1}+k_{2}+k_{3} \geq n+p+2 \\ \left.-L^{-(p+1)}\left(a_{1} \wedge a_{2}\right) \wedge a_{3}\right] & \text { with } k_{1}, k_{2}, k_{3} \leq n+p \\ 0 & \text { otherwise }\end{array}\right.$

The higher $m^{l}$ operations. We set $m^{l}=0$ for all $l \geq 4$.

From [16], we have the following:

Theorem 2.5 (Section 5 of [16]). For any symplectic manifold $M$ of dimension $2 n$ and any integer $p$ with $0 \leq p \leq n$, the operations $m^{l}$ endow $\mathcal{F}_{p}(M)$ with the structure of an $A_{\infty}$-algebra.

\section{Equivalence of algebras}

\subsection{Definition of $\mathcal{C}$}

Let $f: X \rightarrow Y$ be a map of cochain complexes. In homological algebra, the natural notion of quotient is the cone, or mapping cone of $f$. It is defined to be the cochain complex

$$
Y \oplus X[1], \quad d(y \oplus x)=d y+f(x) \oplus-d x .
$$


Finally, a commutative differential graded algebra, or cdga, is a commutative algebra in the category of cochain complexes. Explicitly, a cdga is a cochain complex $A=(A, d)$ together with a map of cochain complexes

$$
m^{2}: A \otimes A \rightarrow A
$$

which is associative, and which satisfies graded commutativity:

$$
m^{2}(a, b)=(-1)^{|a||b|} m^{2}(b, a) .
$$

Note that $m^{2}$ is a map of cochain complexes if and only if it satisfies the Leibniz rule:

$$
d m^{2}(a, b)=m^{2}(d a, b)+(-1)^{|a|} m^{2}(a, d b) .
$$

A map of cdgas is a map of cochain complexes $f: A \rightarrow B$ such that $f \circ m^{2}=$ $m^{2} \circ f \otimes f$.

Remark 3.1. Given any commutative ring $R$, and an element $x$, one has the map of $R$-modules

$$
R \stackrel{x}{\rightarrow} R
$$

and one can define the quotient $R$-module $R / x R$; this also has a commutative ring structure. In fact, the two-term complex above-often known as the Koszul complex of $x$-is a derived enhancement of the quotient module $R / x R$. In what follows, we consider the case of $R=\Omega^{\bullet}(M)$, the de Rham complex of differential forms on a symplectic manifold $M$. Our element $x$ is some power of $\omega \cdot{ }^{1}$ So, heuristically, $\mathcal{C}$ should be thought of as a derived enhancement of " $R / x R$."

Consider the degree 0 map

$$
\omega^{p+1}: \Omega(M)[-2 p-2] \stackrel{\wedge \omega^{p+1}}{\longrightarrow} \Omega(M) .
$$

Then Cone $\left(\omega^{p}\right)$ has in degree $k$ the vector space

$$
\Omega^{k}(M) \oplus \theta \Omega^{k-2 p-1}(M)
$$

where $\theta$ is a formal element of degree $2 p+1$. This has differential

$$
d_{\mathcal{C}}(\eta \oplus \theta \xi)=\left(d \eta+\omega^{p+1} \xi\right) \oplus-\theta d \xi .
$$

This cochain complex has another description endowing it with an obvious cdga structure:

\footnotetext{
${ }^{1}$ In fact, the discussion holds for any even-degree, closed differential form on $M$.
} 
Definition 3.2. We define $\mathcal{C}$ to be the cdga obtained from $\Omega(M)$ by freely attaching a degree $2 p+1$ variable $\theta$ satisfying $d \theta-\omega^{p+1}=0$. Note that $\omega$ and $p$ are implicit in writing $\mathcal{C}$.

Remark 3.3. The variable $\theta$ is a formal degree $2 p+1$ variable, and we are imposing the relation $d \theta=\omega^{p+1}$ in the cone cdga; in particular, we do not make any exactness assumption on $\omega^{p+1}$ as a form on $M$.

Thinking of the cdga $\mathcal{C}$ as an $A_{\infty}$-algebra, we will let $m_{\mathcal{C}}^{1}$ and $m_{\mathcal{C}}^{2}$ denote its differential and its multiplication, respectively, while $m_{\mathcal{C}}^{k}=0$ for all $k \geq 3$. Explicitly:

$m_{\mathcal{C}}^{2}\left(\left(\eta \oplus \theta \eta^{\prime}\right) \otimes\left(\xi \oplus \theta \xi^{\prime}\right)\right)=\left(\eta \oplus \theta \eta^{\prime}\right) \wedge\left(\xi \oplus \theta \xi^{\prime}\right)=\eta \wedge \xi \oplus \theta\left(\eta^{\prime} \wedge \xi+(-1)^{\eta} \eta \wedge \xi^{\prime}\right)$.

\section{2. $\mathcal{C}$ is equivalent to $\mathcal{F}$}

Throughout this section we fix an integer $0 \leq p \leq n$. The dependence of $\mathcal{C}$ and $\mathcal{F}$ on this choice is implicit. Furthermore, the indices $j$ and $k$ take integral values $j \in\{0,1, \ldots, 2 n+2 p+1\}$ and $k \in\{0,1, \ldots, n+p\}$.

We shall write any $p$-filtered form $\alpha \in F^{p} \Omega^{k}$ as

$$
\alpha_{k}=\beta_{k}+\omega \beta_{k-2}+\ldots+\omega^{p} \beta_{k-2 p}
$$

where each $\beta_{i} \in P^{i}$ is a primitive form of degree $i$.

Let us first make the observation that

$$
\begin{aligned}
\mathcal{C}^{k} & =\Omega^{k} \oplus \theta \Omega^{k-2 p-1} \\
\mathcal{C}^{2 n+2 p+1-k} & =\Omega^{2 n+2 p+1-k} \oplus \theta \Omega^{2 n-k}=\left(*_{r} \Omega^{k-2 p-1}\right) \oplus \theta\left(*_{r} \Omega^{k}\right)
\end{aligned}
$$

making use of the relation $*_{r} \Omega^{k}(M)=\Omega^{2 n-k}(M)$ for any integer $k$. The above implies $\mathcal{C}^{k} \cong \mathcal{C}^{2 n+2 p+1-k}$ as expected. Following this, we can write for any $C_{k} \in \mathcal{C}^{k}$

$$
C_{k}=\left(\alpha_{k}+\omega^{p+1} \beta_{k-2 p-2}+\ldots\right)+\theta_{2 p+1}\left(\alpha_{k-2 p-1}+\omega^{p+1} \beta_{k-4 p-3}+\ldots\right),
$$

and for any $C_{2 n+2 p+1-k} \in \mathcal{C}^{2 n+2 p+1-k}$

$$
\begin{aligned}
C_{2 n+2 p+1-k}=*_{r}\left(\alpha_{k-2 p-1}+\right. & \left.\omega^{p+1} \beta_{k-4 p-3}+\ldots\right) \\
& +\theta_{2 p+1} *_{r}\left(\alpha_{k}+\omega^{p+1} \beta_{k-2 p-2}+\ldots\right) .
\end{aligned}
$$


If we further replace the $\alpha_{k-2 p-1}$ terms in (10)-(11) above with its Lefschetz decomposed form

$$
\alpha_{k-2 p-1}=\beta_{k-2 p-1}+\omega \beta_{k-2 p-3}+\ldots+\omega^{p} \beta_{k-2 p},
$$

then we find the relation

$$
\mathcal{C}^{k} \cong \mathcal{C}^{2 n+2 p+1-k} \cong\left(\bigoplus_{0 \leq j \leq k-2 p-1} P^{j}\right) \oplus F^{p} \Omega^{k}
$$

Therefore, the maps

$$
\begin{aligned}
C_{k} & \mapsto\left(\beta_{0}, \ldots, \beta_{k-2 p-1}, \alpha_{k}\right) \\
C_{2 n+2 p+1-k} & \mapsto\left(\beta_{0}, \ldots, \beta_{k-2 p-1}, \alpha_{k}\right)
\end{aligned}
$$

are isomorphisms of $\mathbb{R}$ vector spaces. This gives another representation of elements in $\mathcal{C}^{j}$.

We now define a pair of maps $(f, g)$ that relates $\mathcal{C}$ and $\mathcal{F}$.

Definition 3.4. In terms of (10)-(11), we define

$$
\begin{aligned}
& f: \mathcal{C}^{j} \rightarrow \quad \mathcal{F}^{j} \\
& C_{j} \mapsto\left\{\begin{array}{cl}
\alpha_{j} & j \leq n+p, \\
-\left(\alpha_{k}+\Pi^{p *} \omega^{p} d \alpha_{k-2 p-1}\right) & j \geq n+p+1, j=2 n+2 p+1-k,
\end{array}\right.
\end{aligned}
$$

where $\Pi^{p *} \omega^{p} d \alpha_{k-2 p-1}=\omega^{p} \partial_{+} \beta_{k-2 p-1}$, and

$$
\begin{aligned}
g: \mathcal{F}^{j} & \rightarrow \quad \mathcal{C}^{j} \\
\alpha_{k} & \mapsto\left\{\begin{array}{cl}
\alpha_{k}-\theta_{2 p+1} L^{-(p+1)} d \alpha_{k} & j \leq n+p, k=j, \\
-\theta_{2 p+1} *_{r} \alpha_{k} & j \geq n+p+1, k=2 n+2 p+1-j,
\end{array}\right.
\end{aligned}
$$

where $L^{-(p+1)} d \alpha_{k}=\partial_{-} \beta_{k-2 p}$.

A pictorial description of the $f$ and $g$ maps is given in Figure 2 and Figure 3, respectively. Alternatively, we can also express the maps in terms of the decomposition in (12)-(13). We can write $f: \mathcal{C}^{j} \rightarrow \mathcal{F}^{j}$ as

$$
f:\left(\beta_{0}, \ldots, \beta_{k-2 p-1}, \alpha_{k}\right) \mapsto \begin{cases}\alpha_{k} & j \leq n+p, k=j \\ -\left(\alpha_{k}+\omega^{p} \partial_{+} \beta_{k-2 p-1}\right) & j \geq n+p+1, \\ & k=2 n+2 p+1-j\end{cases}
$$




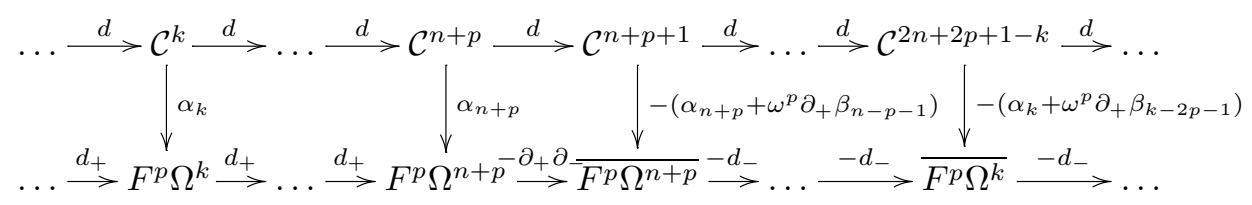

Figure 2: The map $f: \mathcal{C} \rightarrow \mathcal{F}$. The maps are expressed in terms of the decomposition in (10)-(11).

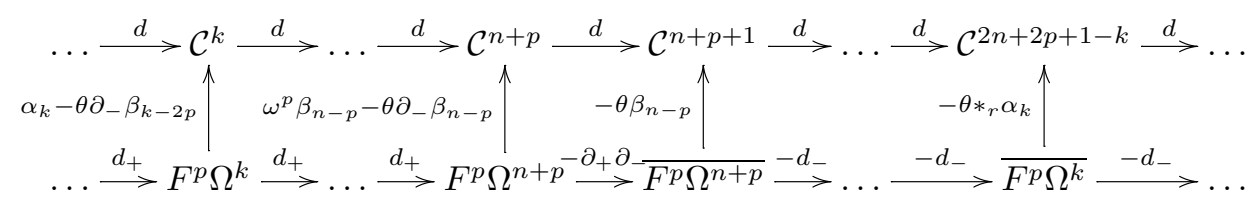

Figure 3: The map $g: \mathcal{F} \rightarrow \mathcal{C}$. In the maps above, we use the notation $\beta_{k-2 p}=L^{-p} \alpha_{k}$, and for the two maps in the middle, we have applied the relation $F^{p} \Omega^{n+p}=\overline{F^{p} \Omega^{n+p}}=L^{p} P^{n-p}$.

and $g: \mathcal{F}^{j} \rightarrow \mathcal{C}^{j}$ as

$$
g: \alpha_{k} \mapsto \begin{cases}\left(0, \ldots,-L^{-(p+1)} d \alpha_{k}, \alpha_{k}\right) & j \leq n+p, k=j \\ \left(0, \ldots, 0,-\alpha_{k}\right) & j \geq n+p+1, k=2 n+2 p+1-j\end{cases}
$$

Lemma 3.5. The maps $f: \mathcal{C} \rightarrow \mathcal{F}$ and $g: \mathcal{F} \rightarrow \mathcal{C}$ are chain maps, i.e.

$$
d_{\mathcal{F}} f=f d_{\mathcal{C}}, \quad d_{\mathcal{C}} g=g d_{\mathcal{F}} .
$$

Proof. The lemma is proved by direct calculation. We first compute

$$
d_{\mathcal{F}} f C_{j}= \begin{cases}d_{+} \alpha_{j} & j<n+p \\ -\omega^{p} \partial_{+} \partial_{-} \beta_{j-2 p} & j=n+p \\ d_{-} \alpha_{k}-\omega^{p} \partial_{+} \partial_{-} \beta_{k-2 p-1} & j=2 n+2 p+1-k>n+p, k \leq n+p\end{cases}
$$

To compute $f d_{\mathcal{C}}$, we consider first the case $j<n+p$. We have

$$
d_{\mathcal{C}} C_{j}=\left(d_{+} \alpha_{j}+\omega^{p+1}\left(\partial_{-} \beta_{j-2 p}+\partial_{+} \beta_{j-2 p-2}\right)+\ldots\right)
$$


Odd sphere bundles, symplectic manifolds \& intersection theory

$$
\begin{aligned}
& +\omega^{p+1}\left(\alpha_{j-2 p-1}+\omega^{p+1} \beta_{j+4 p-3}+\ldots\right) \\
& -\theta_{2 p+1} d\left(\alpha_{j-2 p-1}+\omega^{p+1} \beta_{j+4 p-3}+\ldots\right)
\end{aligned}
$$

It then follows that $f d_{\mathcal{C}} C_{j}=d_{+} \alpha_{j}$. When $j=n+p$, the above equation becomes

$$
\begin{gathered}
\left.d_{\mathcal{C}} C_{n+p}=\omega^{p+1}\left(\partial_{-} \beta_{n-p}+\partial_{+} \beta_{n-p-2}+\beta_{n-p-1}\right)+\mathcal{O}(\omega) \ldots\right) \\
-\theta_{2 p+1} d\left(\alpha_{n-p-1}+\omega^{p+1} \beta_{n+3 p-3}+\ldots\right)
\end{gathered}
$$

Notice that $\theta_{2 p+1} d \alpha_{n-p-1}=\theta_{2 p+1}\left(\partial_{+} \beta_{n-p-1}+\mathcal{O}(\omega)\right)=\theta_{2 p+1} *_{r}\left(\omega^{p} \partial_{+} \beta_{n-p-1}+\right.$ $\left.\mathcal{O}\left(\omega^{p+1}\right)\right)$. Hence, we obtain

$f d_{\mathcal{C}} C_{n+p}=-\left[-\omega^{p} \partial_{+} \beta_{n-p-1}+\omega^{p} \partial_{+}\left(\partial_{-} \beta_{n-p}+\beta_{n-p-1}\right)\right]=-\omega^{p} \partial_{+} \partial_{-} \beta_{n-p}$.

Now for $j>n+p$, let $j=2 n+2 p+1-k$ where $0 \leq k \leq n+p$. Then

$$
\begin{aligned}
d_{\mathcal{C}} C_{j}=\omega^{n+2 p+2-k}\left(\partial_{-} \beta_{k-2 p-1}+\partial_{+} \beta_{k-2 p-3}+\beta_{k-2 p-2}+\mathcal{O}(\omega)\right) & \\
-\theta_{2 p+1} L^{n-k+1} & {\left[\left(\partial_{-} \beta_{k}+\partial_{+} \beta_{k-2}\right)+\ldots\right.} \\
& \left.-\omega^{p}\left(\partial_{-} \beta_{k-2 p}+\partial_{+} \beta_{k-2 p-2}\right)+\ldots\right]
\end{aligned}
$$

This gives the desired result

$$
\begin{aligned}
f d_{\mathcal{C}} C_{2 n+2 p+1-k} & =-\left(-d_{-} \alpha_{k}-\omega^{p} \partial_{+} \beta_{k-2 p-2}+\omega^{p} \partial_{+}\left(\partial_{-} \beta_{k-2 p-1}+\beta_{k-2 p-2}\right)\right. \\
& =d_{-} \alpha_{k}-\omega^{p} \partial_{+} \partial_{-} \beta_{k-2 p-1}
\end{aligned}
$$

The calculation for the $g$ map is straightforward. It can be easily checked that for $\alpha_{k} \in \mathcal{F}^{k}$,

$$
d_{\mathcal{C}} g\left(\alpha_{k}\right)=d_{+} \alpha_{k}+\theta_{2 p+1} \partial_{+} \partial_{-} \beta_{k-2 p}=g d_{\mathcal{F}}\left(\alpha_{k}\right),
$$

and for $\alpha_{k} \in \mathcal{F}^{2 n+2 p+1-k}$,

$$
d_{\mathcal{C}} g\left(\alpha_{k}\right)=\theta_{2 p+1} *_{r} d_{-} \bar{\alpha}_{k}=g d_{\mathcal{F}}\left(\bar{\alpha}_{k}\right)
$$

It is straightforward to check that $f g=\mathrm{id}_{\mathcal{F}}$. Hence, we may wonder whether $g f$ admits a chain homotopy to the identity on $\mathcal{C}$.

Definition 3.6. We define $G: \mathcal{C}^{j} \rightarrow \mathcal{C}^{j-1}$ as follows. For any $\eta \in \Omega^{j}$ and $\xi \in \Omega^{j-2 p-1}$, let

$$
G(\eta+\theta \wedge \xi):=L^{p} \xi+\theta L^{-(p+1)} \eta
$$


In terms of the decomposition (12)-(13), $G: \mathcal{C}^{j} \rightarrow \mathcal{C}^{j-1}$ acts by sending the element $\left(\beta_{0}, \ldots, \beta_{k-2 p-1}, \alpha_{k}\right)$ to

$$
\begin{cases}\left(\beta_{0}, \ldots, \beta_{k-2 p-2}, \omega^{p} \beta_{k-2 p-1}\right) & j \leq n+p, k=j \\ \left(\beta_{0}, \ldots, \beta_{k-2 p-1}, \alpha_{k}\right) & j=n+p+1, k=n+p \\ \left(\beta_{0}, \ldots, \beta_{k-2 p-1}, L^{-p} \alpha_{k}, 0\right) & j>n+p+1, k=2 n+2 p+1-j\end{cases}
$$

where by convention $\beta_{-1}=0$.

Making use of $G$, we have the following result:

Lemma 3.7. The inclusion $g: \mathcal{F} \rightarrow \mathcal{C}$ and the maps $f$ and $G$ exhibit $\mathcal{F}$ as a strong deformation retract of $\mathcal{C}$ :

$$
f g=\operatorname{id}_{\mathcal{F}}, \quad \operatorname{id}_{\mathcal{C}}-g f=d_{\mathcal{C}} G+G d_{\mathcal{C}}
$$

In particular, both $f$ and $g$ are quasi-isomorphisms and $H^{*}(\mathcal{F}) \cong H^{*}(\mathcal{C})$.

Proof of Lemma 3.7. As mentioned, the proof of the first relation $f g=\mathrm{id}_{\mathcal{F}}$ follows directly from the definition of the maps. For the second relation, consider first for $\mathcal{C}^{j=k}$ with $0 \leq k \leq n+p$. For the lefthand side, we have

$$
\begin{aligned}
(g f) C_{k} & =\alpha_{k}-\theta_{2 p+1} \partial_{-} \beta_{k-2 p} \\
\left(\operatorname{id}_{\mathcal{C}}-g f\right) C_{k}= & \left(\omega^{p+1} \beta_{k-2 p-2}+\ldots\right) \\
& \quad+\theta_{2 p+1}\left(\partial_{-} \beta_{k-2 p}+\alpha_{k-2 p-1}+\omega^{p+1} \beta_{k-4 p-3}+\ldots\right)
\end{aligned}
$$

For the righthand side, we have

$$
\begin{aligned}
G C_{k}= & \omega^{p}\left(\alpha_{k-2 p-1}+\omega^{p+1} \beta_{k-4 p-3}+\ldots\right)+\theta_{2 p+1}\left(\beta_{k-2 p-2}+\omega \beta_{k-2 p-4}+\ldots\right) \\
d_{\mathcal{C}} G C_{k}= & \omega^{p} d\left(\alpha_{k-2 p-1}+\omega^{p+1} \beta_{k-4 p-3}+\ldots\right)+\omega^{p+1}\left(\beta_{k-2 p-2}+\omega \beta_{k-2 p-4}+\ldots\right) \\
& \quad-\theta_{2 p+1} d\left(\beta_{k-2 p-2}+\ldots\right) \\
G d_{\mathcal{C}} C_{k}= & -\omega^{p} d\left(\alpha_{k-2 p-1}+\omega^{p+1} \beta_{k+4 p-3}+\ldots\right) \\
& +\theta_{2 p+1}\left[\partial_{-} \beta_{k-2 p}+d\left(\beta_{k-2 p-2}+\ldots\right)+\left(\alpha_{k-2 p-1}+\omega^{p+1} \beta_{k+4 p-3}+\ldots\right)\right]
\end{aligned}
$$

which results in

$$
\begin{aligned}
\left(d_{\mathcal{C}} G+G d_{\mathcal{C}}\right) C_{k}=\omega^{p+1}\left(\beta_{k-2 p-2}+\omega \beta_{k-2 p-4}+\ldots\right) & \\
& +\theta_{2 p+1}\left(\partial_{-} \beta_{k-2 p}+\alpha_{k-2 p-1}+\omega^{p+1} \beta_{k+4 p-3}+\ldots\right)
\end{aligned}
$$

which matches exactly with the lefthand side. 
We now check the case for $\mathcal{C}^{j=2 n+2 p+1-k}$ with $0 \leq k \leq n+p$. For the lefthand side, we have

$$
\begin{aligned}
g f C_{2 n+2 p+1-k}= & \theta_{2 p+1} *_{r}\left(\alpha_{k}+\omega^{p} \partial_{+} \beta_{k-2 p-1}\right) \\
\left(\operatorname{id}_{\mathcal{C}}-g f\right) C_{2 n+2 p+1-k}= & \omega^{n+2 p+1-k}\left(\alpha_{k-2 p-1}+\omega^{p+1} \beta_{k-4 p-3}+\ldots\right) \\
& +\theta_{2 p+1} *_{r}\left(-\omega^{p} \partial_{+} \beta_{k-2 p-1}+\omega^{p+1} \beta_{k-2 p-2}+\ldots\right)
\end{aligned}
$$

For the righthand side, we have

$$
\begin{aligned}
G C_{2 n+2 p+1-k}= & \omega^{n-k+p}\left(\alpha_{k}+\omega^{p+1} \beta_{k-2 p-2}+\ldots\right) \\
& +\theta_{2 p+1} \omega^{n-p+k}\left(\alpha_{k-2 p-1}+\omega^{p+1} \beta_{k-4 p-3}+\ldots\right) \\
d_{\mathcal{C}} G C_{2 n+2 p+1-k}= & \omega^{n-k+p} d\left(\alpha_{k}+\omega^{p+1} \beta_{k-2 p-2}+\ldots\right) \\
& +\omega^{n-k+2 p+1}\left(\alpha_{k-2 p-1}+\omega^{p+1} \beta_{k-4 p-3}+\ldots\right) \\
& -\theta_{2 p+1} \omega^{n+p-k} d\left(\alpha_{k-2 p-1}+\omega^{p+1} \beta_{k-4 p-3}+\ldots\right) \\
d_{\mathcal{C}} C_{2 n+2 p+1-k}= & \omega^{n+2 p+1-k}\left[d\left(\alpha_{k-2 p-1}+\omega^{p+1} \beta_{k-4 p-3}+\ldots\right)-\partial_{+} \beta_{k-2 p-1}\right] \\
& +\omega^{n-k+p+1}\left(\omega^{p+1} \beta_{k-2 p-2}+\ldots\right) \\
& -\theta_{2 p+1} \omega^{n-k} d\left(\alpha_{k}+\omega^{p+1} \beta_{k-2 p-2}+\ldots\right) \\
G d_{\mathcal{C}} C_{2 n+2 p+1-k}= & -\omega^{n-k+p} d\left(\alpha_{k}+\omega^{p+1} \beta_{k-2 p-2}+\ldots\right) \\
& +\theta_{2 p+1} \omega^{n-k+p+1}\left(\beta_{k-2 p-2}+\ldots\right) \\
& +\theta_{2 p+1} \omega^{n+p-k}\left[d\left(\alpha_{k-2 p-1}+\omega^{p+1} \beta_{k-4 p-3}+\ldots\right)\right.
\end{aligned}
$$

Note that in the righthand side of the third equation, we have used the property that $L^{p+1} *_{r} \alpha_{k}=0$ and also added the term $-\omega^{n+2 p+1-k} \partial_{+} \beta_{k-2 p-1}$ since it is identically zero. The above equations give the result

$$
\begin{aligned}
d_{\mathcal{C}} G+G d_{\mathcal{C}}= & \omega^{n-k+2 p+1}\left(\alpha_{k-2 p-1}+\omega^{p+1} \beta_{k-4 p-3}+\ldots\right) \\
& +\theta_{2 p+1} \omega^{n-k}\left[-\omega^{p} \partial_{+} \beta_{k-2 p-1}+\left(\omega^{p+1} \beta_{k-2 p-2}+\ldots\right)\right]
\end{aligned}
$$

which is exactly the lefthand side.

Though $f$ and $g$ are chain maps, they are clearly not compatible with the product structure of the algebras $\mathcal{C}$ and $\mathcal{F}$. For instance, it is not difficult to see that for generic $a_{1}, a_{2} \in \mathcal{F}, g m_{\mathcal{F}}^{2}\left(a_{1} \otimes a_{2}\right) \neq m_{\mathcal{C}}^{2}\left(g a_{1} \otimes g a_{2}\right)$. So we treat the cdga $\mathcal{C}$ as an $A_{\infty}$-algebra. Recall that a sequence of maps 
$g^{l}: \mathcal{F}^{\otimes l} \rightarrow \mathcal{C}[1-l]$ with $l=1,2, \ldots$ is called an $A_{\infty}$ map if the following equations are satisfied (see for example Section 3.4 of [12]):

$$
\sum_{r+s+t=n}(-1)^{r+s t} g^{l}\left(1^{\otimes r} \otimes m_{\mathcal{F}}^{s} \otimes 1^{\otimes t}\right)=\sum_{i_{1}+\ldots+i_{q}=n}(-1)^{u} m_{\mathcal{C}}^{q}\left(g^{i_{1}} \otimes \ldots g^{i_{q}}\right), n \geq 1
$$

where $l=r+1+t$ and the sign on the right-hand side is given by

$$
u=(q-1)\left(i_{1}-1\right)+(q-2)\left(i_{2}-1\right)+\ldots+2\left(i_{q-2}-1\right)+\left(i_{q-1}-1\right) .
$$

Theorem 3.8. The sequence of maps $g^{l}: \mathcal{F}^{\otimes l} \rightarrow \mathcal{C}[1-l]$ given by

1. $g^{1}=g$,

2. $g^{2}=-\theta_{2 p+1} L^{-(p+1)} m_{\mathcal{C}}^{2}\left(g^{1} \otimes g^{1}\right)$,

3. $g^{l}=0$ for all $l \geq 3$.

defines an $A_{\infty}$-algebra map $\mathcal{F} \rightarrow \mathcal{C}$.

We will give the proof of this theorem in the next subsection. For now, we observe the following:

Proof of Theorem 1.1. Since the map $g$ is an quasi-isomorphism, Theorem 3.8 gives the equivalence $\mathcal{F}_{p} \simeq \operatorname{Cone}\left(\omega^{p+1}\right)$ as $A_{\infty}$-algebras. Moreover, one has an equivalence of cdgas $\operatorname{Cone}\left(\omega^{p+1}\right) \simeq \Omega\left(E_{p}\right)$ from Theorem A.1 of the Appendix, which of course is also an equivalence of $A_{\infty}$-algebras. Now it remains to prove that the maps $g^{l}$ are natural with respect to immersions $f: M \rightarrow M^{\prime}$ with $\operatorname{dim} M=\operatorname{dim} M^{\prime}$ and $f^{*} \omega^{\prime}=\omega$. This is clear for the definition of $g=g^{1}$ in Definition 3.4, which is compatible with pull-back of forms, and likewise for $g^{2}$ as defined in Theorem 3.8. (Note that the dimension restriction and the $f^{*} \omega^{\prime}=\omega$ condition guarantee that $f^{*}\left(L^{\prime} \alpha\right)=L f^{*} \alpha$, and likewise $f^{*}\left(*_{r}^{\prime} \alpha\right)=*_{r} f^{*}(\alpha)$.)

\subsection{Proof of Theorem 3.8}

For the proof, we will make use of a few useful formulas gather together in Lemma 3.9. Below, the bracket [,] denotes the standard graded commutator.

Lemma 3.9. Let $\eta$, $\xi$ be forms on $M$ with $|\xi|=|\eta|-(2 p+1)$. The following hold: 
Odd sphere bundles, symplectic manifolds \& intersection theory 233

1.

$$
\begin{aligned}
& {\left[m_{\mathcal{C}}^{1}, \theta L^{-(p+1)}\right](\eta+\theta \xi)=} \\
& \omega^{p+1} L^{-(p+1)} \eta-\theta\left(\left[d, L^{-(p+1)}\right] \eta-L^{-(p+1)} \omega^{p+1} \xi\right) .
\end{aligned}
$$

2.

$$
\left[d, L^{-(p+1)}\right] \eta= \begin{cases}-L^{(p+1)} d \Pi^{p} \eta & |\eta| \leq n+p \\ \Pi^{p *} d L^{-(p+1)} \eta & |\eta|>n+p\end{cases}
$$

3. The $g^{2}$ operation on any $a_{1}, a_{2} \in \mathcal{F}$ can be expressed as

$$
g^{2}\left(a_{1}, a_{2}\right)= \begin{cases}-\theta L^{-(p+1)}\left(a_{1} \wedge a_{2}\right) & \left|a_{1}\right|,\left|a_{2}\right|<n+p \\ 0 & \text { otherwise }\end{cases}
$$

Proof of Lemma 3.9. (1): We have

$$
m_{\mathcal{C}}^{1}\left(\theta L^{-(p+1)}\right)(\eta+\theta \xi)=m_{\mathcal{C}}^{1}\left(\theta L^{-(p+1)} \eta\right)=\omega L^{-(p+1)} \eta-\theta d L^{-(p+1)} \eta
$$

and

$$
\begin{aligned}
\theta L^{-(p+1)} m_{\mathcal{C}}^{1}(\eta+\theta \xi) & =\theta L^{-(p+1)}(d \eta+\omega \xi-\theta d \xi) \\
& =\theta\left(L^{-(p+1)} d \eta+L^{-(p+1)} \omega \xi\right) .
\end{aligned}
$$

The result follows.

(2): Decomposing

$$
\eta=\alpha_{k}+\omega^{p+1} \beta_{k-2 p-2}+\ldots
$$

we have that

$d L^{-(p+1)} \eta=d\left(\beta_{k-2 p-2}+\ldots\right)=\partial_{+} \beta_{k-2 p-2}+\omega\left(\partial_{-} \beta_{k-2 p-2}+\partial_{+} \beta_{k-2 p-4}\right)+\ldots$

while

$$
L^{-(p+1)} d \eta=L^{-(p+1)}\left(\ldots+\omega^{p+1}\left(\partial_{-} \beta_{k-2 p}+\partial_{+} \beta_{k-2 p-2}\right)+\ldots\right)
$$

so we have

$$
\left[d, L^{-(p+1)}\right] \eta=-\partial_{-} \beta_{k-2 p}
$$

which is equal to $-L^{-(p+1)} d \Pi^{p} \eta$ by definition of $\Pi^{p}$. 
When $|\eta|>n+p$, let $|\eta|=n+p+r$, with $r>0$. Then,

$$
\begin{aligned}
\left(d L^{-(p+1)}-\right. & \left.L^{-(p+1)} d\right) \eta \\
= & \left(d L^{-(p+1)}-L^{-(p+1)} d\right)\left(\omega^{p+r} \beta_{n-p-r}+\omega^{p+r+1} \beta_{n-p-r-2}+\ldots\right) \\
= & d\left(\omega^{r-1} \beta_{n-p-r}+\omega^{r} \beta_{n-p-r-2}+\ldots\right) \\
& \quad-L^{-(p+1)}\left(\omega^{p+r+1}\left(\partial_{-} \beta_{n-p-r}+\partial_{+} \beta_{n-p-r-2}\right)+\ldots\right) \\
= & \omega^{r-1} \partial_{+} \beta_{n-p-r}
\end{aligned}
$$

which is what we claimed.

(3): By definition, we have that $g^{2}=-\theta L^{-(p+1)} m_{\mathcal{C}}^{2}\left(g^{1} \otimes g^{1}\right)$. Note that if any term of $m_{\mathcal{C}}^{2}\left(g^{1} \otimes g^{1}\right)$ has a factor of $\theta$ in it, the operation $\theta L^{-(p+1)}$ renders it zero since $\theta \wedge \theta=0$ in $\mathcal{C}$. On the other hand, $g^{1} a$ for $a \in \mathcal{F}$ has a term without a $\theta$ only when $|a|<n+p$.

Proof of Theorem 3.8. We need to show that the sequence of maps $g^{l}$ : $\mathcal{F}^{\otimes l} \rightarrow \mathcal{C}[1-l]$ satisfy the $A_{\infty}$ map condition of $(17)$

$\sum_{r+s+t=n}(-1)^{r+s t} g^{l}\left(1^{\otimes r} \otimes m_{\mathcal{F}}^{s} \otimes 1^{\otimes t}\right)=\sum_{i_{1}+\ldots+i_{q}=n}(-1)^{u} m_{\mathcal{C}}^{q}\left(g^{i_{1}} \otimes \ldots g^{i_{q}}\right), n \geq 1$

where $l=r+1+t$ and the sign on the right-hand side is given by

$$
u=(q-1)\left(i_{1}-1\right)+(q-2)\left(i_{2}-1\right)+\ldots+2\left(i_{q-2}-1\right)+\left(i_{q-1}-1\right) .
$$

Noting that $m_{\mathcal{C}}^{k}=0$ for $k \geq 3$ and $m_{\mathcal{F}}^{k}=0$ for $k \geq 4$, and further, that $g^{k}=0$ for $k \geq 3$, (17) becomes the following four equations:

$(\mathrm{n}=1)$

$$
m_{\mathcal{C}}^{1} g^{1}=g^{1} m_{\mathcal{F}}^{1}
$$

$(\mathrm{n}=2)$

$$
m_{\mathcal{C}}^{1} g^{2}+g^{2}\left(m_{\mathcal{F}}^{1} \otimes 1+1 \otimes m_{\mathcal{F}}^{1}\right)+m_{\mathcal{C}}^{2}\left(g^{1} \otimes g^{1}\right)=g^{1} m_{\mathcal{F}}^{2}
$$

$$
g^{2}\left(1 \otimes m_{\mathcal{F}}^{2}-m_{\mathcal{F}}^{2} \otimes 1\right)+m_{\mathcal{C}}^{2}\left(g^{1} \otimes g^{2}-g^{2} \otimes g^{1}\right)=g^{1} m_{\mathcal{F}}^{3}
$$

$(\mathrm{n}=4)$

$$
g^{2}\left(1 \otimes m_{\mathcal{F}}^{3}+m_{\mathcal{F}}^{3} \otimes 1\right)+m_{\mathcal{C}}^{2}\left(g^{2} \otimes g^{2}\right)=0 .
$$

The $n=1$ equation. We verified $g=g^{1}$ is a chain map in the proof of Lemma 3.5.

The $n=2$ equation. We first simplify the expression involving $g^{2}$ terms:

$$
\begin{aligned}
& m_{\mathcal{C}}^{1} g^{2}+g^{2}\left(m_{\mathcal{F}}^{1} \otimes 1+1 \otimes m_{\mathcal{F}}^{1}\right) \\
& =m_{\mathcal{C}}^{1}\left(-\theta L^{-(p+1)} m_{\mathcal{C}}^{2}\left(g^{1} \otimes g^{1}\right)\right)+\left(-\theta L^{-(p+1)} m_{\mathcal{C}}^{2}\left(g^{1} \otimes g^{1}\right)\right)\left(m_{\mathcal{F}}^{1} \otimes 1+1 \otimes m_{\mathcal{F}}^{1}\right)
\end{aligned}
$$




$$
\begin{aligned}
& =-m_{\mathcal{C}}^{1}\left(\theta L^{-(p+1)}\right) m_{\mathcal{C}}^{2}\left(g^{1} \otimes g^{1}\right)+\left(-\theta L^{-(p+1)} m_{\mathcal{C}}^{2}\right)\left(m_{\mathcal{C}}^{1} \otimes 1+1 \otimes m_{\mathcal{C}}^{1}\right)\left(g^{1} \otimes g^{1}\right) \\
& =-m_{\mathcal{C}}^{1}\left(\theta L^{-(p+1)}\right) m_{\mathcal{C}}^{2}\left(g^{1} \otimes g^{1}\right)-\left(\theta L^{-(p+1)} m_{\mathcal{C}}^{1} m_{\mathcal{C}}^{2}\right)\left(g^{1} \otimes g^{1}\right) \\
& =-\left(m_{\mathcal{C}}^{1}\left(\theta L^{-(p+1)}\right)+\theta L^{-(p+1)} m_{\mathcal{C}}^{1}\right) m_{\mathcal{C}}^{2}\left(g^{1} \otimes g^{1}\right) \\
& =-\left[m_{\mathcal{C}}^{1}, \theta L^{-(p+1)}\right] m_{\mathcal{C}}^{2}\left(g^{1} \otimes g^{1}\right) .
\end{aligned}
$$

We have used that $g^{1}$ is a chain map, and that the operations $m_{\mathcal{C}}^{1}$ and $m_{\mathcal{C}}^{2}$ satisfy the Leibniz rule. So the left-hand side of the $n=2$ equation becomes

$$
\begin{aligned}
m_{\mathcal{C}}^{1} g^{2}+g^{2}\left(m_{\mathcal{F}}^{1} \otimes 1+1 \otimes m_{\mathcal{F}}^{1}\right)+m_{\mathcal{C}}^{2}\left(g^{1} \otimes g^{1}\right) \\
=\left(-\left[m_{\mathcal{C}}^{1}, \theta L^{-(p+1)}\right]+1\right) m_{\mathcal{C}}^{2}\left(g^{1} \otimes g^{1}\right) .
\end{aligned}
$$

Let $a_{1}, a_{2}$ be elements of $\mathcal{F}$. Let us write

$$
m_{\mathcal{C}}^{2}\left(g^{1} \otimes g^{1}\right)\left(a_{1} \otimes a_{2}\right)=\eta+\theta \xi .
$$

Then, by Lemma 3.9(1), we find

$$
\begin{aligned}
\left(m_{\mathcal{C}}^{1} g^{2}+\right. & \left.g^{2}\left(m_{\mathcal{F}}^{1} \otimes 1+1 \otimes m_{\mathcal{F}}^{1}\right)+m_{\mathcal{C}}^{2}\left(g^{1} \otimes g^{1}\right)\right)\left(a_{1} \otimes a_{2}\right) \\
& =\left(-\left[m_{\mathcal{C}}^{1}, \theta L^{-(p+1)}\right]+1\right)(\eta+\theta \xi) \\
& =\left(1-\omega^{p+1} L^{-(p+1)}\right) \eta+\theta\left(\left[d, L^{-(p+1)}\right] \eta+\left(1-L^{-(p+1)} \omega^{p+1}\right) \xi\right) \\
& =\Pi^{p} \eta+\theta\left(\left[d, L^{-(p+1)}\right] \eta+\Pi^{p *} \xi\right) .
\end{aligned}
$$

The expression for $\eta$ and $\xi$ are found by calculating $m_{\mathcal{C}}^{2}\left(g^{1} \otimes g^{1}\right)\left(a_{1} \otimes a_{2}\right)$ explicitly. Recalling the definitions of $g^{1}$ in Definition 3.4, we find that $m_{\mathcal{C}}^{2}\left(g^{1} \otimes g^{1}\right)\left(a_{1} \otimes a_{2}\right)$ equals

$$
\begin{cases}a_{1} \wedge a_{2}-\theta\left(L^{-(p+1)} d a_{1} \wedge a_{2}+(-1)^{a_{1}} a_{1} \wedge L^{-(p+1)} d a_{2}\right) & \left|a_{1}\right|,\left|a_{2}\right| \leq n+p \\ -\theta\left((-1)^{a_{1}} a_{1} \wedge *_{r} a_{2}\right) & \left|a_{1}\right| \leq n+p,\left|a_{2}\right|>n+p \\ -\theta\left(*_{r} a_{1}\right) \wedge a_{2} & \left|a_{1}\right|>n+p,\left|a_{2}\right| \leq n+p \\ 0 & \left|a_{1}\right|,\left|a_{2}\right|>n+p .\end{cases}
$$

We can now read off the expression for $\eta$ and $\xi$ and substitute them into (20). In the case of $\left|a_{1}\right|,\left|a_{2}\right| \leq n$, we have

$$
\eta=a_{1} \wedge a_{2}, \quad \xi=-L^{-(p+1)} d a_{1} \wedge a_{2}-(-1)^{a_{1}} a_{1} \wedge L^{-(p+1)} d a_{2} .
$$


Using Lemma 3.9(2), we find in this case that

$$
\begin{aligned}
& \Pi^{p} \eta+\theta\left(\left[d, L^{-(p+1)}\right] \eta+\Pi^{p *} \xi\right) \\
& = \begin{cases}\Pi^{p}\left(a_{1} \wedge a_{2}\right)-L^{-(p+1)} d \Pi^{p}\left(a_{1} \wedge a_{2}\right) & \left|a_{1}\right|+\left|a_{2}\right| \leq n+p \\
\theta \Pi^{p *}\left[d L^{-(p+1)}\left(a_{1} \wedge a_{2}\right)\right. & \left|a_{1}\right|+\left|a_{2}\right|>n+p \\
\left.-\left(L^{-(p+1)} d a_{1}\right) \wedge a_{2}-(-1)^{a_{1}} a_{1} \wedge L^{-(p+1)} d a_{2}\right] & \end{cases}
\end{aligned}
$$

The other three cases in (20) are straightforward. All in all, we find that the left-hand side of the $n=2$ condition matches exactly the right-hand side (using the definition of $m_{\mathcal{F}}^{2}$ in Section 2.3) given by

$$
\begin{aligned}
& g^{1} m_{\mathcal{F}}^{2}\left(a_{1} \otimes a_{2}\right) \\
& = \begin{cases}\Pi^{p}\left(a_{1} \wedge a_{2}\right)-\theta L^{-(p+1)} d \Pi^{p}\left(a_{1} \wedge a_{2}\right) & \left|a_{1}\right|+\left|a_{2}\right| \leq n+p \\
-\theta \Pi^{p *}\left(-d L^{-(p+1)}\left(a_{1} \wedge a_{2}\right)\right. & \left|a_{1}\right|+\left|a_{2}\right|>n+p \\
\left.\quad+L^{-(p+1)} d a_{1} \wedge a_{2}+(-1)^{a_{1}} a_{1} \wedge L^{-(p+1)} d a_{2}\right) & \text { with }\left|a_{1}\right|,\left|a_{2}\right| \leq n+p \\
-\theta \Pi^{p *}\left((-1)^{a_{1}} a_{1} \wedge *_{r} a_{2}\right) & \left|a_{1}\right| \leq n+p,\left|a_{2}\right|>n+p \\
-\theta \Pi^{p *}\left({ }_{r} a_{1} \wedge a_{2}\right) & \left|a_{1}\right|>n+p,\left|a_{2}\right| \leq n+p \\
0 & \left|a_{1}\right|,\left|a_{2}\right|>n+p\end{cases}
\end{aligned}
$$

The $n=3$ equation. Let $a_{1}, a_{2}, a_{3}$ be elements of $\mathcal{F}$. Consider first the left-hand side of the equation:

$$
\left[g^{2}\left(1 \otimes m_{\mathcal{F}}^{2}-m_{\mathcal{F}}^{2} \otimes 1\right)+m_{\mathcal{C}}^{2}\left(g^{1} \otimes g^{2}-g^{2} \otimes g^{1}\right)\right]\left(a_{1} \otimes a_{2} \otimes a_{3}\right) .
$$

Notice that each term vanishes unless $\left|a_{1}\right|,\left|a_{2}\right|,\left|a_{3}\right| \leq n+p$. For the first term, this is due to Lemma 3.9(3). For the second term, since the $g^{2}$ map contains a $\theta$, a $\theta$ in the $g^{1}$ map term would result in a null $m_{\mathcal{C}}^{2}$. This forces the element which $g^{1}$ acts on to have degree less than or equal to $n+p$. Hence, we only need to consider the case where $\left|a_{1}\right|,\left|a_{2}\right|,\left|a_{3}\right| \leq n+p$. We note that this is a necessary condition for $m_{\mathcal{F}}^{3}\left(a_{1} \otimes a_{2} \otimes a_{3}\right)$ to be non-trivial.

For the first term, we have

$$
\begin{aligned}
g^{2} & \left(1 \otimes m_{\mathcal{F}}^{2}-m_{\mathcal{F}}^{2} \otimes 1\right)\left(a_{1} \otimes a_{2} \otimes a_{3}\right) \\
& =-\theta L^{-(p+1)} m_{\mathcal{C}}^{2}\left[a_{1} \otimes\left(m_{\mathcal{F}}^{2}\left(a_{2} \otimes a_{3}\right)\right)-m_{\mathcal{F}}^{2}\left(a_{1} \otimes a_{2}\right) \otimes a_{3}\right] \\
& =-\theta L^{-(p+1)}\left[a_{1} \wedge \Pi^{p}\left(a_{2} \wedge a_{3}\right)-\Pi^{p}\left(a_{1} \wedge a_{2}\right) \wedge a_{3}\right] \\
& =-\theta L^{-(p+1)} L^{p+1}\left[a_{1} \wedge L^{-(p+1)}\left(a_{2} \wedge a_{3}\right)-L^{-(p+1)}\left(a_{1} \wedge a_{2}\right) \wedge a_{3}\right]
\end{aligned}
$$


where in the third line, we omitted the term including $\theta$ in the $m_{\mathcal{F}}^{2}$, and in the third line, we used the identity $\Pi^{p}=1-L^{p+1} L^{-(p+1)}$.

For the second term, we have

$$
\begin{aligned}
& m_{\mathcal{C}}^{2}\left(g^{1} \otimes g^{2}-g^{2} \otimes g^{1}\right)\left(a_{1} \otimes a_{2} \otimes a_{3}\right) \\
& =m_{\mathcal{C}}^{2}\left[g^{1} \otimes\left(-\theta L^{-(p+1)}\right) m_{\mathcal{C}}^{2}\left(g^{1} \otimes g^{1}\right)\right. \\
& \left.\quad-\left(-\theta L^{-(p+1)}\right) m_{\mathcal{C}}^{2}\left(g^{1} \otimes g^{1}\right) \otimes g^{1}\right]\left(a_{1} \otimes a_{2} \otimes a_{3}\right) \\
& =-\theta\left[a_{1} \wedge L^{-(p+1)}\left(a_{2} \wedge a_{3}\right)-L^{-(p+1)}\left(a_{1} \wedge a_{2}\right) \wedge a_{3}\right]
\end{aligned}
$$

Combining the two expressions, we find for the left-hand side of the equation

$$
\begin{aligned}
& {\left[g^{2}\left(1 \otimes m_{\mathcal{F}}^{2}-m_{\mathcal{F}}^{2} \otimes 1\right)+m_{\mathcal{C}}^{2}\left(g^{1} \otimes g^{2}-g^{2} \otimes g^{1}\right)\right]\left(a_{1} \otimes a_{2} \otimes a_{3}\right)} \\
& =-\theta\left(1-L^{-(p+1)} L^{p+1}\right)\left[a_{1} \wedge L^{-(p+1)}\left(a_{2} \wedge a_{3}\right)-L^{-(p+1)}\left(a_{1} \wedge a_{2}\right) \wedge a_{3}\right] \\
& =-\theta \Pi^{p *}\left[a_{1} \wedge L^{-(p+1)}\left(a_{2} \wedge a_{3}\right)-L^{-(p+1)}\left(a_{1} \wedge a_{2}\right) \wedge a_{3}\right] \\
& =g^{1} m_{\mathcal{F}}^{3}\left(a_{1} \otimes a_{2} \otimes a_{3}\right)
\end{aligned}
$$

The $n=4$ equation. This equation is satisfied because every term is identically zero. To wit:

- $g^{2}\left(1 \otimes m_{\mathcal{F}}^{3}+m_{\mathcal{F}}^{3} \otimes 1\right)$ is zero for the following reason: $m_{\mathcal{F}}^{3}$ is non-zero only when $m_{\mathcal{F}}^{3}$ outputs an element of degree $\geq n+p+1$. Thus, by Lemma 3.9(3), $g^{2}\left(1 \otimes m_{\mathcal{F}}^{3}\right)$ and $g^{2}\left(m_{\mathcal{F}}^{3} \otimes 1\right)$ are both identically zero.

- By definition, the output of $g^{2}$ involves a $\theta$, an odd degree form. Thus, $m_{\mathcal{C}}^{2}\left(g^{2} \otimes g^{2}\right)$ vanishes since it involves the wedge product of $\theta$ with itself.

Notation: To simplify notation, we will also refer the family of $A_{\infty}$ maps $\left\{g^{l}\right\}$ as $g$ in the sections following.

\section{Calabi-Yau structure on $\mathcal{F}$}

What we call a Calabi-Yau structure follows the terminology of [4], though this notion has also been called a proper Calabi-Yau structure [7], a right Calabi-Yau structure [3], and a "symplectic structure on a formal pointed dg-manifold" [13]. ${ }^{2}$

\footnotetext{
${ }^{2}$ This is to contrast it with a different notion of Calabi-Yau-ness, which has been called a smooth Calabi-Yau structure [7] and a left Calabi-Yau structure [3]
} 
Definition 4.1. An $A_{\infty}$-algebra $A$ over a base ring $k$, together with a bilinear map

$$
\langle,\rangle: A \otimes A \rightarrow k[-D]
$$

is called Calabi-Yau of dimension $D$ if

- The pairing is non-degenerate on cohomology,

- $\langle a, b\rangle=(-1)^{|a||b|}\langle b, a\rangle$, and

- $\langle$,$\rangle satisfies the cyclic symmetry equation$

$$
\left\langle m^{l}\left(a_{1}, \ldots, a_{l}\right), a_{l+1}\right\rangle=(-1)^{l+\left|a_{1}\right|\left(\left|a_{2}\right|+\ldots+\left|a_{l+1}\right|\right)}\left\langle m^{l}\left(a_{2}, \ldots, a_{l+1}\right), a_{1}\right\rangle .
$$

Example 4.2. Let $X$ be a compact oriented manifold of dimension $D$. Then the cdga $\Omega(X)$ can be given the structure of a Calabi-Yau algebra of dimension $D$ by the following pairing:

$$
\eta \otimes \xi \mapsto \int_{X} \eta \wedge \xi
$$

Note that if $\eta$ and $\xi$ do not have complementary degree, the integral vanishes.

Remark 4.3. Calabi-Yau algebras were first defined by Kontsevich to formalize properties of $D^{b}$ Coh of a smooth Calabi-Yau variety. It is a theorem of Costello [4] that Calabi-Yau algebras, and Calabi-Yau categories more generally, define a topological CFT. For further discussion, see also Ginzburg [8], and the work of Brav-Dyckerhoff [3] generalizing the notion to relative Calabi-Yau structures.

If our symplectic manifold $M$ is compact and $[\omega]$ is an integral class in cohomology, then $\mathcal{C}$ is also a Calabi-Yau algebra. This is because the mapping cone Cone $\left(\omega^{p+1}\right)$ can be identified (as a cdga) with the minimal model for the the cdga $\Omega\left(E_{p}\right)$ - the differential forms on the total space $E_{p}$ of the sphere bundle associated to $\omega^{p+1}$. (See Theorem A.1.) Furthermore, since $\omega$ is symplectic, $E_{p}$ comes with an orientation. If $M$ is compact, so is $E$, hence Example 4.2 implies that $\Omega\left(E_{p}\right) \simeq \mathcal{C}$ is Calabi-Yau.

The algebra equivalence of $\mathcal{C}$ and $\mathcal{F}$ however holds for all $\omega$ regardless of its integrality. Since the Calabi-Yau property is algebraic in nature, it is

elsewhere. A category or algebra may be smoothly Calabi-Yau but not properly Calabi-Yau and vice versa; the distinction is important, for instance, as these different structures gives rise to two-dimensional field theories that are only partially defined, and the class of cobordisms on which the field theories are defined differs by the type of Calabi-Yau-ness one equips onto the category. We refer the reader to Section 6 of [7] for a more thorough account. 
reasonable to expect that whether $\mathcal{F}$ exhibits the Calabi-Yau property or not should not depend on the integrality of $[\omega]$. Indeed, we can prove directly on $M$ that $\mathcal{F}$ is a Calabi-Yau algebra for all compact $\left(M^{2 n}, \omega\right)$.

To define the pairing on $\mathcal{F}$, we make use of the map $\mathcal{F}_{p} \rightarrow \mathbb{R}[-2(n+$ $p)-1)$ ] given by

$$
\overline{F^{p} \Omega^{0}} \stackrel{*_{r}}{\longrightarrow} \Omega^{2 n}(M) \stackrel{\int_{M}}{\longrightarrow} \mathbb{R} .
$$

For degree reasons, this map is zero on all other degree components of $\mathcal{F}$. The map gives us a natural pairing for $\mathcal{F}$.

Definition 4.4 (The Calabi-Yau structure on $\mathcal{F}$ ). Define the pairing on $\mathcal{F}$ by the composition

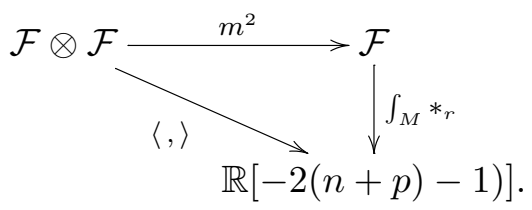

Explicitly, for $a_{1} \in F^{p} \Omega^{k}$ and $a_{2} \in \overline{F^{p} \Omega^{k}}$,

$$
\left\langle a_{1}, a_{2}\right\rangle=\int_{M} *_{r}\left[m^{2}\left(a_{1}, a_{2}\right)\right]=\int_{M}(-1)^{k} a_{1} \wedge *_{r} a_{2}=\int_{M} *_{r} a_{2} \wedge a_{1}=\left\langle a_{2}, a_{1}\right\rangle .
$$

Remark 4.5. Note that $m^{2}$ is already graded-commutative, so the second condition of Definition 4.1 is automatically satisfied. It is also worth noting that if $a_{1} \in F^{p} \Omega^{k_{1}}$ and $a_{2} \in \overline{F^{p} \Omega^{k_{2}}}$, we must have that $k_{2}=2 n+2 p+1-$ $k_{1}$ if $\left\langle a_{1}, a_{2}\right\rangle$ is non-zero. In this case, $\left|a_{1}\right|\left|a_{2}\right|=0 \bmod 2$, and therefore, $\left\langle a_{1}, a_{2}\right\rangle=\left\langle a_{2}, a_{1}\right\rangle$ as consistent with (23).

The pairing (23) defined on $\mathcal{F}$ is in fact compatible to the Calabi-Yau pairing $\langle,\rangle_{\mathcal{C}}$ on $\mathcal{C}$ induced from $\Omega(E)$ when $\omega$ is integral. In this case, we have the following commutative diagram:

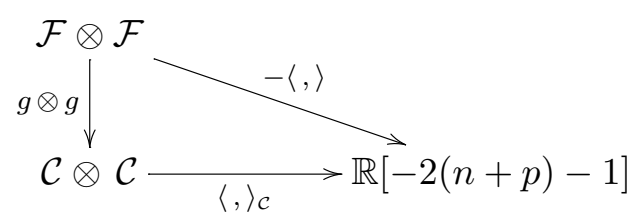

To verify this, consider $\alpha_{k} \in \mathcal{F}^{k}$ and $\alpha_{k}^{\prime} \in \mathcal{F}^{2 n+2 p+1-k}$ of complementary degrees. Recalling the definition of the chain map $g$ in Definition 3.4, we have 


$$
\begin{aligned}
\left\langle g \alpha_{k}, g \alpha_{k}^{\prime}\right\rangle_{\mathcal{C}} & =\int_{E_{p}}\left(\alpha_{k}-\theta_{2 p+1} L^{-(p+1)} d \alpha_{k}\right) \wedge\left(-\theta_{2 p+1} *_{r} \alpha_{k}^{\prime}\right) \\
& =-\int_{E_{p}} \theta_{2 p+1} \wedge(-1)^{k} \alpha_{k} \wedge *_{r} \alpha_{k}^{\prime} \\
& =-\int_{M}(-1)^{k} \alpha_{k} \wedge *_{r} \alpha_{k}^{\prime}=-\left\langle\alpha_{k}, \alpha_{k}^{\prime}\right\rangle
\end{aligned}
$$

where in the last second line, we noted that the equivalence of Cone $\left(\omega^{p+1}\right) \simeq$ $\Omega\left(E_{p}\right)$ is compatible with the Thom isomorphism for differential forms; namely, $\theta$ represents the Thom class, and for any differential form $A$ on $M$,

$$
\int_{E_{p}} \theta_{2 p+1} \wedge A=\int_{M} A
$$

We now show that pairing on $\mathcal{F}$ satisfy the Calabi-Yau algebra conditions for any symplectic structure.

Theorem 4.6. Definition 4.4 endows $\mathcal{F}_{p}{ }^{\bullet}$ with a Calabi-Yau structure of dimension $2(n+p)+1$.

Proof. Non-degeneracy. The non-degeneracy of the pairing (23) on cohomology was previously shown in two special cases: (i) for $p=0$ and $k \neq n$ in Proposition 3.7 of [18]; (ii) for $k=n+p$ in Proposition 3.26 of [17]. We will give the argument here for the remaining cases of $k<n+p$ (which follow the same techniques as the previous cases).

We make use of a compatible triple, $(\omega, J, g)$, introducing a compatible almost complex structure, $J$, and the associated Riemannian metric, $g$, on $\left(M^{2 n}, \omega\right)$. Since the filtered cohomologies $F^{p} H(M)$ are associated with elliptic complexes (see Theorem 2.4), there is an elliptic Laplacian associated with each cohomology. By Hodge theory, there then exists a unique harmonic representative for each cohomology class. The harmonic representative for $k<n+p$ satisfies the following:

$$
\begin{array}{ll}
d_{+} a_{1}=0, & d_{-} a_{2}=0, \\
d_{+}^{*} a_{1}=0, & d_{-}^{*} a_{2}=0 .
\end{array}
$$

for $a_{1} \in F^{p} \Omega^{k}$ and $a_{2} \in \overline{F^{p} \Omega^{k}}$ and the adjoint here is defined with respect to the standard inner product on differential forms

$$
\left(\eta_{k}, \xi_{k}\right)=\int_{M} \eta_{k} \wedge * \xi_{k}
$$


where $\eta_{k}, \xi_{k} \in \Omega^{k}(M)$ and, $*$, the Hodge star operator. Let us recall that the action of the Hodge star on the Lefschetz decomposed element, $\omega^{r} \beta_{s}$, where $\beta_{s} \in P^{s}(M)$, is given by (see for example [17])

$$
* \omega^{r} \beta_{s}=(-1)^{s(s+1) / 2} \frac{r !}{(n-r-s) !} \omega^{n-r-s} \mathcal{J} \beta_{s}
$$

where

$$
\mathcal{J}=\sum_{p, q}(\sqrt{-1})^{p-q} \Pi^{p, q}
$$

with $\Pi^{p, q}$ defined to be the projection of a form into its $(p, q)$ components with respect to the almost complex structure $J$. Introducing further the operator $\mathcal{R}$ defined to be

$$
\mathcal{R}\left(\omega^{r} \beta_{s}\right):=(-1)^{s(s+1) / 2} \frac{r !}{(n-r-s) !} \omega^{r} \beta_{s},
$$

we then have the relation

$$
*={ }_{r} \mathcal{R} \mathcal{J} \text {. }
$$

We can now express $\left\{d_{+}, d_{-}\right\}$and their adjoints acting on the space of $p$ filtered forms, $F^{p} \Omega(M)$, as follows:

$$
\begin{array}{ll}
d_{+}=\Pi^{p} d, & d_{-}=*_{r} d *_{r}, \\
d_{+}^{*}=-* d *, & d_{-}^{*}=-\mathcal{R} \mathcal{J} d \mathcal{R} \mathcal{J} .
\end{array}
$$

Now, if $a_{1} \in F^{p} \Omega^{k}(M)$ is a harmonic representative of $F^{p} H_{+}^{k}(M)$, then $d_{-}\left(\mathcal{R} \mathcal{J} a_{1}\right)=*_{r} d * a_{1}=0$, that is, $\mathcal{R} \mathcal{J}\left(a_{1}\right)$ represents a cohomology class of $F^{p} H_{-}^{k}(M)$. Similarly, if $a_{2} \in \overline{F^{p} \Omega^{k}}(M)$ is a harmonic representative of $F^{p} H_{-}^{k}(M)$, then $\mathcal{R} \mathcal{J}\left(a_{2}\right)$ represents a cohomology class of $F^{p} H_{+}^{k}(M)$. Finally, to prove non-degeneracy of the pairing (23) on cohomology, choose $a_{1}$ to be a non-trivial harmonic representative of $F^{p} H_{+}^{k}(M)$. Then setting $a_{2}=\mathcal{R} \mathcal{J} a_{1}$, we have

$$
\left\langle a_{1}, a_{2}\right\rangle=\int_{M}(-1)^{k} a_{1} \wedge *_{r} a_{2}=(-1)^{k} \int_{M} a_{1} \wedge *_{r} \mathcal{R} \mathcal{J} a_{1}=(-1)^{k}\left\|a_{1}\right\|^{2} \neq 0,
$$


having applied (25). Similarly, for $a_{2}$ a non-trivial harmonic representative of $F^{p} H_{-}^{k}(M)$, we take $a_{1}=\mathcal{R} \mathcal{J} a_{2}$ and this results in

$$
\left\langle a_{2}, a_{1}\right\rangle=\int_{M}{ }^{*} a_{2} \wedge a_{1}=\int_{M} *_{r} a_{2} \wedge \mathcal{R} \mathcal{J} a_{2}=\int_{M} a_{2} \wedge *_{r} \mathcal{R} \mathcal{J} a_{2}=\left\|a_{2}\right\|^{2} \neq 0,
$$

thereby proving the non-degeneracy of the pairing on $F^{p} H_{ \pm}^{k}(M)$ for $k<$ $n+p$.

Symmetry. This is Remark 4.5.

Cyclic symmetry for $l=1$. There are two cases that we need to consider. First, fix $a_{1} \in F^{p} \Omega^{k-1}, a_{2} \in \overline{F^{p} \Omega^{k}}$ and let $k \leq n+p$. Then the cyclic condition (22) becomes:

$$
\begin{aligned}
\left\langle m^{1} a_{1}, a_{2}\right\rangle & =\int_{M} *_{r} a_{2} \wedge d_{+} a_{1} \\
& =\int_{M} *_{r} a_{2} \wedge d a_{1} \\
& =(-1)^{k+1} \int_{M} d *_{r} a_{2} \wedge a_{1} \\
& =(-1)^{k} \int_{M} *_{r}\left(-d_{-}\right) a_{2} \wedge a_{1}=(-1)^{1+\left|a_{1}\right|\left|a_{2}\right|}\left\langle m^{1} a_{2}, a_{1}\right\rangle
\end{aligned}
$$

Here, (26) holds because $d_{+} a_{1}=\Pi^{p} d a_{1}=d a_{1}-L^{p+1} L^{-(p+1)} d a_{1}$, and since $a_{2}$ is a $p$-filtered form, $L^{p+1} *_{r} a_{2}=0$. (27) follows from integration by parts. Note also that $\left|a_{2}\right|=2(n+p)+1-k$ and therefore $\left|a_{1}\right|\left|a_{2}\right|=k \bmod 2$.

For the second case, let $a_{1}, a_{2} \in F^{p} \Omega^{n+p}$. Then,

$$
\begin{aligned}
& \left\langle m^{1} a_{1}, a_{2}\right\rangle=\int_{M} *_{r}(-d \Lambda d) a_{1} \wedge a_{2}=\int_{M}(-d \Lambda d) a_{1} \wedge *_{r} a_{2} \\
& =(-1)^{n+p+1} \int_{M} \Lambda d a_{1} \wedge d *_{r} a_{2}=(-1)^{n+p+1} \int_{M} d a_{1} \wedge \Lambda d *_{r} a_{2} \\
& =\int_{M} a_{1} \wedge d \Lambda d *_{r} a_{2}=(-1)^{1+(n+p)^{2}} \int_{M} *_{r}(-d \Lambda d) a_{2} \wedge a_{1} \\
& =(-1)^{1+\left|a_{1}\right|\left|a_{2}\right|}\left\langle m^{1} a_{2}, a_{1}\right\rangle
\end{aligned}
$$

where we have noted that $\partial_{+} \partial_{-}=d \Lambda d$ which commutes with $*_{r}$, and also, for any $\eta \in \Omega^{k+2}$ and $\eta^{\prime} \in \Omega^{2 n-k}$,

$$
\int_{M} \Lambda \eta \wedge \eta^{\prime}=\int_{M} \eta \wedge \Lambda \eta^{\prime}
$$


Cyclic symmetry for $l=2$. The relation is

$$
\begin{aligned}
\left\langle m^{2}\left(a_{1}, a_{2}\right), a_{3}\right\rangle & =(-1)^{\left|a_{1}\right|\left(\left|a_{2}\right|+\left|a_{3}\right|\right)}\left\langle m^{2}\left(a_{2}, a_{3}\right), a_{1}\right\rangle \\
& =\left\langle a_{1}, m^{2}\left(a_{2}, a_{3}\right)\right\rangle
\end{aligned}
$$

Hence, we need to show

$$
\int_{M} *_{r}\left[m^{2}\left(a_{1}, m^{2}\left(a_{2}, a_{3}\right)\right)-m^{2}\left(m^{2}\left(a_{1}, a_{2}\right), a_{3}\right)\right]=0
$$

for $\left|a_{1}\right|+\left|a_{2}\right|+\left|a_{3}\right|=2 n+2 p+1$. This follows from integrating the $A_{\infty}$ relations for $m^{3}$. Explicitly, the $A_{\infty}$ relation relevant here is $m^{2}\left(1 \otimes m^{2}\right)-m^{2}\left(m^{2} \otimes 1\right)=m^{1}\left(m^{3}\right)+m^{3}\left(m^{1} \otimes 1 \otimes 1+1 \otimes m^{1} \otimes 1+1 \otimes 1 \otimes m^{1}\right)$.

Plugging in elements $a_{1}, a_{2}, a_{3}$, the terms involving $m^{2}$ on the left-hand side are precisely those in (29). So we can show instead that $\int_{M}{ }_{M}\left\{\left[m^{1}\left(m^{3}\right)+m^{3}\left(m^{1} \otimes 1 \otimes 1+1 \otimes m^{1} \otimes 1+1 \otimes 1 \otimes m^{1}\right)\right]\left(a_{1} \otimes a_{2} \otimes a_{3}\right)\right\}=0$. Notice that the first term vanishes since $m^{3}\left(a_{1}, a_{2}, a_{3}\right) \in \overline{F^{p} \Omega^{1}}$ and therefore

$$
\begin{aligned}
\int_{M} *_{r}\left[m^{1} m^{3}\left(a_{1}, a_{2}, a_{3}\right)\right] & =-\int_{M} *_{r} d_{-}\left[m^{3}\left(a_{1}, a_{2}, a_{3}\right)\right] \\
& =-\int_{M} d\left[{ }_{r} m^{3}\left(a_{1}, a_{2}, a_{3}\right)\right]=0
\end{aligned}
$$

by Stokes's Theorem. For the remaining terms, the only possible non-trivial contribution of the integrand takes the form

$$
\begin{aligned}
& d_{+} a_{1} \wedge L^{-(p+1)}\left(a_{2} \wedge a_{3}\right)-L^{-(p+1)}\left(d_{+} a_{1} \wedge a_{2}\right) \wedge a_{3} \\
& +(-1)^{i}\left[a_{1} \wedge L^{-(p+1)}\left(d_{+} a_{2} \wedge a_{3}\right)-L^{-(p+1)}\left(a_{1} \wedge d_{+} a_{2}\right) \wedge a_{3}\right] \\
& +(-1)^{i+j}\left[a_{1} \wedge L^{-(p+1)}\left(a_{2} \wedge d_{+} a_{3}\right)-L^{-(p+1)}\left(a_{1} \wedge a_{2}\right) \wedge d_{+} a_{3}\right]
\end{aligned}
$$

where $\left|a_{1}\right|,\left|a_{2}\right|,\left|a_{3}\right|<n+p$. In fact, each term above is zero. For instance, consider the first term in (30). To take account of the $L^{-(p+1)}$ operator, we can express

$$
\alpha_{j} \wedge \alpha_{k}=\omega^{n+2 p+1-i} \wedge \eta_{i-2 p-1}
$$


where $\eta_{i-2 p-1} \in \Omega^{i-2 p-1}$ and $\alpha_{l} \in F^{p} \Omega^{l}$. In above, we have used the fact that $i+j+k=2 n+1$ and $i<n+p$. Then, we have

$$
d_{+} \alpha_{i} \wedge L^{-(p+1)}\left(\alpha_{j} \wedge \alpha_{k}\right)=\omega^{n+p-i} \wedge d_{+} \alpha_{i} \wedge \eta_{i-2 p-1}=0
$$

by the $p$-filter condition $L^{n+p-i}\left(F^{p} \Omega^{i+1}\right)=0$. Similar computations for the other terms show that all the terms in (30) vanish identically.

Cyclic symmetry for $l=3$. This follows directly from the definition of $m^{3}$ and applying (28). Since $m^{l}=0$ for $l \geq 4$, this finishes the proof.

Remark 4.7. To any $A_{\infty}$-algebra with a Calabi-Yau structure, one can associate a two-dimensional topological conformal field theory [4]. The action of this field theory consists of a standard kinetic term and a potential term $\Phi$. The potential term is determined by the $A_{\infty}$ structure and takes the form (see, for example [10])

$$
\Phi(x)=\sum_{l=1}^{\infty} \frac{1}{l+1}\left\langle m^{l}(x, x, \ldots, x), x\right\rangle,
$$

where $x$ is a formal sum of elements of the $A_{\infty}$-algebra. For instance, for the de Rham cdga in dimension $D=3$, with $x=\alpha_{1}, \Phi\left(x=\alpha_{1}\right)$ is the Chern-Simon functional

$$
\Phi(x)=\frac{1}{2}\left\langle d \alpha_{1}, \alpha_{1}\right\rangle+\frac{1}{3}\left\langle\alpha_{1} \wedge \alpha_{1}, \alpha_{1}\right\rangle .
$$

It is also straightforward to write down the potential for $\mathcal{F}$. For example, in dimension four and with $x=\beta_{1}+\beta_{2}$, where $\beta_{i} \in F^{0} \Omega^{i}(M)$, one finds

$$
\Phi(x)=\left\langle m^{2}\left(\beta_{2}, \beta_{2}\right), \beta_{1}\right\rangle+\frac{1}{2}\left\langle m^{3}\left(\beta_{1}, \beta_{1}, \beta_{2}\right), \beta_{2}\right\rangle .
$$

As far as we are aware, such a field theory with primitive forms as fields has not heretofore appeared in the literature.

\section{Homology and intersection when $\omega$ is integral}

In this section, we will assume $\omega \in H^{2}(M, \mathbb{Z})$ so that $E_{p}$ is a smooth manifold. On $E_{p}$, there is the standard homology and intersection theory on it. The equivalence of $\Omega\left(E_{p}\right) \simeq \mathcal{F}_{p}(M)$ suggests that there should also be a dual homology and intersection theory on $(M, \omega)$, and one that is distinctively symplectic in nature. Here, we will provide a description of what such a theory should look like on $M$. For simplicity, we will focus on the $p=0$ case, 
and in this case, the objects involved turn out to be coisotropic and isotropic spaces on $M$. Due to its close association with forms, we will use the language of currents to describe these spaces. Admittedly, our description here is somewhat heuristic in nature and will require further investigations which we plan to pursue in the future.

Since $p$ will be taken to be zero for the most part, we will drop the subscript label in $E_{p}$ and $\mathcal{F}_{p}$ for the rest of this section.

\subsection{Recollections on currents}

Recall that the collection $\mathcal{D}_{m}$ of $m$-dimensional currents is defined to be the weak $\mathbb{R}$-linear dual to the space of compactly supported smooth $m$-forms on a manifold of dimension $d$. Given a current $\rho$, its boundary $\partial \rho$ is defined by dualizing the de Rham differential:

$$
\partial \rho(\eta):=\rho(d \eta) .
$$

A form of the de Rham theorem (see, for example [9]) states that the resulting chain complex of currents

$$
0 \rightarrow \mathcal{D}_{d} \stackrel{\partial}{\rightarrow} \mathcal{D}_{d-1} \stackrel{\partial}{\rightarrow} \ldots \stackrel{\partial}{\rightarrow} \mathcal{D}_{0} \rightarrow 0 \ldots
$$

has homology isomorphic to singular homology, provided that the manifold $M$ is connected, compact, and oriented:

$$
H_{*}(\mathcal{D}) \cong H_{*}(M ; \mathbb{R}) .
$$

Remark 5.1. Since $\mathcal{D}_{m}$ is a very large vector space, one can often restrict to smaller subspaces while still retaining the same homology. For example, one can show that currents that are represented by Lipschitz neighborhood retracts, and whose $\partial$ are also represented by such, have the same cohomology. (This follows by observing that both theories satisfy the Eilenberg-Steenrod axioms; see for instance Federer [6].)

Example 5.2. Note that oriented, compact $m$-manifolds $X \subset M$ (possibly with boundary) define distributions by sending $\eta \mapsto \int_{X} \eta$. We also note that, when $M$ is compact and oriented of dimension $d$, any $(d-m)$-form $\rho$ defines a linear map by $\eta \mapsto \int_{M} \eta \wedge \rho$.

Indeed, given any $X$, one can define its dual current to be represented by a distributional form $\rho_{X}$ such that

$$
\int_{X} \eta=\int_{M} \eta \wedge \rho_{X}
$$


Here, $\rho_{X}$ is a differential form with coefficients in distributions; the distribution coefficient is the Dirac delta function supported on $X$.

\subsection{Homology of primitive currents}

Extending to currents the definition of a primitive form defined in Section 2.1 , we will call a current primitive if it vanishes under the interior product with the Poisson bivector field.. Coisotropic and isotropic subspaces are related to primitive currents by the following:

Lemma 5.3 (Section 4 of [17]). Let $N \subset M$ be an embedded, compact, oriented submanifold with dual current $\rho_{N}$. Then

(i) $\rho_{N}$ is primitive if and only if $N$ is coisotropic;

(ii) $*_{r} \rho_{N}$ is primitive if and only if $N$ is isotropic.

To see how coisotropic and isotropic subspaces both arise in the $p=0$ case, we take the cochain complex of (9)

$$
\mathcal{F}=\left(P^{0} \stackrel{\partial_{+}}{\longrightarrow} P^{1} \stackrel{\partial_{+}}{\longrightarrow} \ldots \stackrel{d_{+}}{\longrightarrow} P^{n} \stackrel{-\partial_{+} \partial_{-}}{\longrightarrow} \bar{P}^{n} \stackrel{-\partial_{-}}{\longrightarrow} \bar{P}^{n-1} \stackrel{-\partial_{-}}{\longrightarrow} \ldots \stackrel{-\partial_{-}}{\longrightarrow} \bar{P}^{0}\right)
$$

and make a modification by replacing the "bar" elements $\bar{F}^{0} \Omega^{k}=\bar{P}^{k}$ by ${ }_{r} P^{k}=\omega^{n-k} P^{k}$. This results in the following complex:

$\tilde{\mathcal{F}}=\left(P^{0} \stackrel{\partial_{+}}{\longrightarrow} P^{1} \stackrel{\partial_{+}}{\longrightarrow} \ldots \stackrel{\partial_{+}}{\longrightarrow} P^{n} \stackrel{-\partial_{+} \partial_{-}}{\longrightarrow} \omega^{0} P^{n} \stackrel{-d}{\longrightarrow} \omega^{1} P^{n-1} \stackrel{-d}{\longrightarrow} \ldots \stackrel{-d}{\longrightarrow} \omega^{n} \bar{P}^{0}\right)$.

Note that $*_{r} \partial_{-} *_{r}=d$, which is just the standard exterior derivative when acting on $\omega^{n-k} P^{k}$ forms.

Following the intuition of Lemma 5.3, the first half of $\tilde{\mathcal{F}}$ can be roughly thought of as dual to a complex of coisotropic chains $C_{\bullet}$; the second half is dual to a complex of isotropic chains $I_{\bullet}$ since $*_{r}\left(\omega^{n-k} P^{k}\right)=P^{k}$. In all, the cochain complex (31) suggests that we seek a putative chain complex of coisotropic and isotropic currents:

$$
C_{2 n} \stackrel{\partial^{\prime}}{\rightarrow} C_{2 n-1} \stackrel{\partial^{\prime}}{\rightarrow} \ldots \stackrel{\partial^{\prime}}{\rightarrow} C_{n} \stackrel{\partial^{\prime \prime}}{\rightarrow} I_{n} \stackrel{\partial}{\rightarrow} I_{n-1} \stackrel{\partial}{\rightarrow} \ldots \stackrel{\partial}{\rightarrow} I_{0} .
$$

Here, $C_{k}$ is the vector space generated by currents supported by coisotropic chains of dimension $k$, while $I_{k}$ is generated by currents supported on isotropic chains of dimension $k$, with $C_{n}=I_{n}=\mathcal{L}$ being the space of Lagrangian currents. The boundary maps $\left\{\partial, \partial^{\prime \prime}, \partial^{\prime}\right\}$ then have the following interpretations: 
1. $\partial$ is dual to the exterior derivative, $d$, and therefore $\partial$ is just the standard boundary map. Note that the boundary of an isotropic subspace is also isotropic.

2. $\partial^{\prime \prime}$ is dual to $\partial_{+} \partial_{-}=d \circ \partial_{-}$. This is a "boundary" operator mapping Lagrangians to Lagrangians. One way to understand $\partial^{\prime \prime}$ is to study first the dual of the operator $\partial_{-}$. Strikingly, the differential operator $\partial_{-}$lowers the degree of a current by one and so its dual would need to increase the dimension of a chain by one. Such a novel operation can be motivated by noting that for a degree $n$ primitive current, $\beta_{n}$, $d \beta_{n}=\omega \wedge\left(\partial_{-} \beta_{n}\right)$, which dualizes to

$$
\partial L=H \cap L_{+},
$$

with $L$ being the Lagrangian chain dual to $\beta_{n}, H$ the $(2 n-2)$-dimensional symplectic submanifold dual to $\omega$, and $L_{+}$being dual to $\partial_{-} \beta_{n} .^{3}$ In words, $L_{+}$is an $(n+1)$-dimensional chain that intersects with $H$ along the boundary of $L$. Altogether, $\partial^{\prime \prime}$ should correspond to the composition of $L \rightarrow L_{+} \rightarrow \partial L_{+}$, i.e. it maps $L$ to the boundary of $L_{+}$.

3. $\partial^{\prime}$ is dual to $\partial_{+}=\Pi^{0} \circ d$ where $\Pi^{0}: \Omega^{k} \rightarrow P^{k}$ is the projection operator onto the primitive component (see (6)). This suggests that $\partial^{\prime}=\pi^{0} \circ \partial$ where $\pi^{0}$ heuristically "projects" onto the coisotropic component of the boundary. For a better intuition, we note that for $\beta_{k}$, a primitive $k$-current, $d \beta_{k}=\partial_{+} \beta_{k}+\omega \wedge\left(\partial_{-} \beta_{k}\right)$. This dualizes to

$$
\partial^{\prime} C=\partial C-H \cap C_{+}
$$

where $C$ is the $(2 n-k)$-dimensional coisotropic chain dual to $\beta_{k}$, and $C_{+}$is the $(2 n-k+1)$-dimensional coisotropic chain dual to $\partial_{-} \beta_{k}$. Hence, the projection $\pi^{0}$ effectively removes point $x \in \partial C$ whose tangent space $T_{x}(\partial C) \subset T_{x} H$.

The chain complex (32) implies several distinct homologies for isotropic and coisotropic chains on $\left(M^{2 n}, \omega\right)$. For isotropic chains, we have

$$
H_{k}^{I}(M):=\frac{\operatorname{ker}\left(\partial: I_{k} \rightarrow I_{k-1}\right)}{\operatorname{im}\left(\partial: I_{k+1} \rightarrow I_{k}\right)}, \quad 0 \leq k \leq n-1,
$$

\footnotetext{
${ }^{3}$ The existence of a symplectic submanifold dual to $[\omega]$ an integral class is currently known only for $[k \omega]$ where $k$ is a sufficiently large integer [5].
} 
associated to the right hand side of (32). This is just standard homology but with objects restricted to isotropic chains. For coisotropics chains on left hand side of (32), we have the homology:

$$
H_{2 n-k}^{C}(M):=\frac{\operatorname{ker}\left(\partial^{\prime}: C_{2 n-k} \rightarrow C_{2 n-k-1}\right)}{\operatorname{im}\left(\partial^{\prime}: C_{2 n-k+1} \rightarrow C_{2 n-k}\right)}, \quad 0 \leq k \leq n-1 .
$$

Of note, coisotropic elements in the homology can have boundary, but the boundary may not have any coisotropic component. Lastly, at the center of chain complex (32), we have two homologies involving Lagrangians:

$$
\begin{aligned}
H_{n}^{I}(M) & :=\frac{\operatorname{ker}\left(\partial: \mathcal{L}_{n} \rightarrow I_{n-1}\right)}{\operatorname{im}\left(\partial^{\prime \prime}: \mathcal{L}_{n} \rightarrow \mathcal{L}_{n}\right)}, \\
H_{n}^{C}(M) & =\frac{\operatorname{ker}\left(\partial^{\prime \prime}: \mathcal{L}_{n} \rightarrow \mathcal{L}_{n}\right)}{\operatorname{im}\left(\partial^{\prime}: C_{n+1} \rightarrow \mathcal{L}_{n}\right)} .
\end{aligned}
$$

These two Lagrangian homologies certainly can have elements that are not in the standard homology $H_{n}(M)$. For $H_{n}^{I}(M)$, though the Lagrangian generators must be boundaryless, a Lagrangian that is the boundary of an $(n+1)$-dimensional chain can be a non-trivial element. Such a Lagrangian would only be trivial in $H_{n}^{I}(M)$ if it is also in the image of the $\partial^{\prime \prime}$ map. On the other hand, for $H_{n}^{C}(M)$, its elements may include Lagrangians which have non-trivial boundary but are trivial under the $\partial^{\prime \prime}$ map. Examples of both cases will be seen in the Kodaira-Thurston four-manifold discussed at the end of this section.

\subsection{Intersection theory via lifts to $E$}

To better understand these homologies, we can make use of the isomorphism between the cohomology of primitive currents, $F^{0} H(M):=P H(M)$, and the singular cohomology of the contact circle bundle, $H\left(E_{0}\right)$. Then we can explicitly pull back the currents in $\tilde{\mathcal{F}}(M)$ to currents in $\Omega(E)$ by reinterpreting the map $g: \mathcal{F}(M) \rightarrow \Omega(E)$ from Definition 3.4 as a map $\tilde{g}: \tilde{\mathcal{F}}(M) \rightarrow \Omega(E)$. See Figure 4.

The interpretation of the map $\tilde{g}$ at the chain level is as follows:

- A coisotropic chain $C_{2 n-k}$ of codimension $k$ in $M$ (represented by the dual current $\beta_{k} \in P^{k}(M)$ ), is mapped to a codimension $k$ chain $\tilde{C}_{2 n+1-k}$ of dimension $(2 n+1-k)$. The extra dimension comes from $\tilde{C}$ wrapping around the circle fiber of $E$ plus another the addition of 


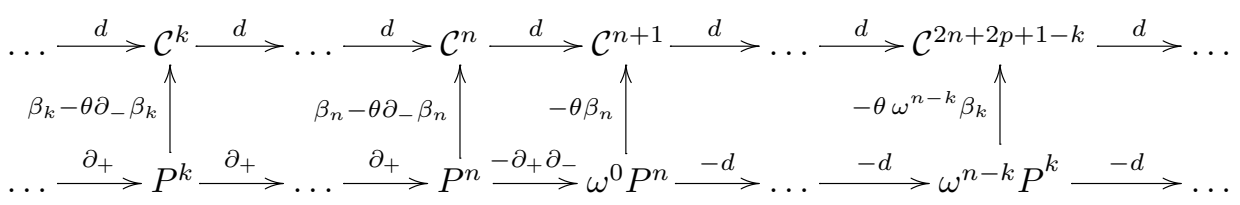

Figure 4: The map $\tilde{g}: \tilde{\mathcal{F}}(M) \rightarrow \Omega(E)$.

another component that is the dual of $\theta \partial_{-} \beta_{k}$. This additional component is important as those coisotropic chains that have non-trivial boundary, but yet the boundary does not have a coisotropic component, become boundaryless cycles after lifted to $E$ (since $\beta_{k}-\theta \partial_{-} \beta_{k}$ is $d$-closed).

- An isotropic chain $I_{k}$ of dimension $k$ in $M$ (represented by the dual current $\omega^{n-k} \beta_{k} \in \omega^{n-k} P^{k}$ ) gets mapped to a section $\tilde{I}_{k}$ of $E$ which is still of dimension $k$. This is possible since $\left.\omega\right|_{I}=0$ and thus the restricted bundle $\left.E\right|_{I}$ is trivial. In particular, if $k=n$, then $I$ is a Lagrangian and the resulting $\tilde{I}$ in $E$ is Legendrian. Furthermore, if $d\left(\omega^{n-k} \beta_{k}\right)=0$, this implies that $I$ has no boundary and neither does $\tilde{I}$ after the lift.

Under the map $\tilde{g}$, the homologies $\left\{H_{\bullet}^{C}(M), H_{\bullet}^{I}(M)\right\}$ map to the standard homology $\left\{H_{\bullet}(E)\right\}$. Thus the Calabi-Yau pairing on $\mathcal{F}$ is interpreted as the usual intersection pairing of $\tilde{C}_{2 n+1-k}$ with $\tilde{I}_{k}$ inside $E$.

\subsection{Example: Kodaira-Thurston four-fold.}

The Kodaira-Thurston manifold, $K T^{4}$, is a closed, non-Kähler, symplectic four-manifold. It can be defined as the quotient of $\mathbb{R}^{4}$ with coordinates $\left\{x_{1}, x_{2}, x_{3}, x_{4}\right\}$ under the identification

$$
\left(x_{1}, x_{2}, x_{3}, x_{4}\right) \sim\left(x_{1}+a, x_{2}+b, x_{3}+c, x_{4}+d-b x_{3}\right)
$$

where $a, b, c, d \in \mathbb{Z}$. On $K T^{4}$, global one-forms can be written as

$$
e_{1}=d x_{1}, \quad e_{2}=d x_{2}, \quad e_{3}=d x_{3}, \quad e_{4}=d x_{4}+x_{2} d x_{3} .
$$

We will take the symplectic structure on $K T^{4}$ to be

$$
\omega=e_{1} \wedge e_{2}+e_{3} \wedge e_{4}
$$




\begin{tabular}{c|c|c|c|c|c|c}
$j$ & 0 & 1 & 2 & 3 & 4 & 5 \\
\hline \multirow{2}{*}{$H^{j}\left(K T^{4}\right)$} & \multirow{2}{*}{1} & $e_{1}$, & $\omega$ & $\omega e_{1}$, & & \\
& & $e_{2}$, & $e_{12}-e_{34}$, & $\omega e_{2}$, & $\omega^{2}$ & \\
& & $e_{3}$ & $e_{13}, e_{24}$ & $\omega e_{4}$ & & \\
\hline \multirow{2}{*}{$F^{0} H^{j}\left(K T^{4}\right)$} & \multirow{2}{*}{1} & $e_{1}$, & $e_{12}-e_{34}$, & $e_{12}-e_{34}$, & $e_{1}$, & \\
& & $e_{2}$, & $e_{13}, e_{24}$, & $e_{13}, e_{24}$, & $e_{2}$, & 1 \\
& & $e_{3}$ & $e_{14}$ & $e_{23}$ & $e_{4}$ & \\
\hline \multirow{2}{*}{$H^{j}(E)$} & \multirow{2}{*}{1} & $e_{1}$, & $e_{12}-e_{34}$, & $\theta\left(e_{12}-e_{34}\right)$, & $\theta\left(\omega e_{1}\right)$, & \multirow{2}{*}{$e_{2}}$, \\
& & $e_{13}, e_{24}$, & $\theta e_{13}, \theta e_{24}$ & $\theta\left(\omega e_{2}\right)$ & $\theta \omega^{2}$ \\
& & $e_{3}$ & $e_{14}+\theta e_{3}$ & $\theta e_{23}$ & $\theta\left(\omega e_{4}\right)$ &
\end{tabular}

Figure 5: Basis of generators for the cohomologies of the Kodaira-Thurston four-manifold $K T^{4}$ and its circle bundle $E$ (where $d \theta=\omega$ ). Here we use the notation $e_{i j}=e_{i} e_{j}$ and $e_{i j k}=e_{i} e_{j} e_{k}$.

It is worthwhile to point out that $K T^{4}$ can be interpreted as a torus bundle over a torus in two ways:
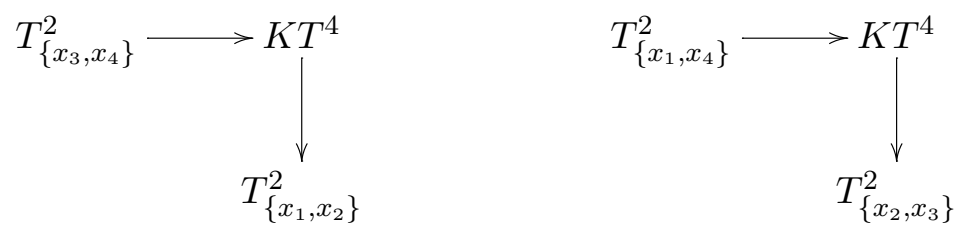

On the left, the fiber torus over a point on the base is a symplectic submanifold with respect to the $\omega$ of (34). In contrast, the fiber torus on the right is a Lagrangian. In fact, it is actually a special Lagrangian with respect to the global $(2,0)$-form $\Omega^{2,0}=\left(e_{1}+i e_{2}\right)\left(e_{3}+i e_{4}\right)$.

To define $E$, the circle bundle over $K T^{4}$, we define the global angular one-form

$$
\theta=d y+x_{1} d x_{2}-x_{4} d x_{3}
$$

where $y$ is the coordinate on $S^{1}=\mathbb{R} / \mathbb{Z}$ with $y \sim y+1$. Clearly, $d \theta=\omega$ on $E$. Moreover, that $\theta$ is globally-defined implies that the circle fiber is twisted over $K T^{4}$ with the identification

$$
\left(x_{1}, x_{4}, y\right) \sim\left(x_{1}+a, x_{4}+d, y-a x_{2}+d x_{3}\right)
$$

where $a, d \in \mathbb{Z}$.

In Table 5, we give a basis of generators for the de Rham cohomology and the $p=0$ filtered cohomology of $K T^{4}$, and also the de Rham cohomol- 
ogy of its circle bundle. They have a heuristic dual chain description when promoted to currents. The two special generators in $F^{0} H^{*}(M)$ that are distinct from those in $H^{*}(M)$ are $e_{14} \in F^{0} H^{2}(M)$ and $e_{23} \in F^{0} H^{3}(M)$. They are both dual to Lagrangians.

1. $e_{14} \in F^{0} H^{2}\left(K T^{4}\right)$ corresponds to the dual current of a Lagrangian $L$ spanning the torus base in the $\left\{x_{2}, x_{3}\right\}$ directions. Note that $d e_{14}=$ $-e_{123}$, hence, the Lagrangian $L$ has a non-trivial boundary, i.e. $\partial L \neq 0$. However, under the lift to $e_{14}+\theta e_{3} \in H^{2}(E)$, the corresponding threedimensional cycle $\tilde{L} \subset E$ has no boundary.

2. $e_{23} \in F^{0} H^{3}\left(K T^{4}\right)$ is the dual current of a Lagrangian $L^{\prime}$ wrapping the torus fiber spanning $\left\{x_{1}, x_{4}\right\}$ over a point on the base. Since $e_{23}=d e_{4}$, $L^{\prime}$ is rationally the boundary of a three-dimensional subspace.

3. Note that the intersection of $L$ and $L^{\prime}$ can be defined on $M$. The value corresponds to the standard intersection (even though $L$ is not a cycle).

\section{Functoriality of $\mathcal{F}$}

Many invariants are useful not just for what they assign to objects, but also for how they behave under morphisms. In this section we prove that the assignment $M \mapsto \mathcal{F}_{p}(M)$ enjoys several functorial properties. First, if $f: M \rightarrow M^{\prime}$ is a symplectic map (so $f^{*} \omega^{\prime}=\omega$ ), the entire sequence of $p$-filtered algebras admits a pullback morphism. Second, if $f_{0}$ and $f_{1}$ are smoothly homotopic by an orthogonal homotopy, the pullback maps are also chain-homotopic. Third, just as $\omega$ is a section of a sheaf on $M$-indeed, because $\omega$ is a section of a sheaf - we prove that the assignment $U \mapsto \mathcal{F}_{p}(U)$ forms a sheaf of $A_{\infty}$-algebras on $M$, and we give an interpretation of the filtered cohomologies as sheaf cohomology. We conclude by showing that any isotropic (in particular, Lagrangian) correspondence between symplectic manifolds induces a bimodule between the filtered algebras of each symplectic manifolds. This bimodule structure is local, in that it factors through the restriction of these algebras to an arbitrarily small neighborhood of the isotropic.

Remark 6.1. The sheaf-theoretic interpretation is useful for the following reason. Until this section, we have treated $\mathcal{F}_{p}(M)$ and Cone $\left(\omega^{p}\right)$ simply as $A_{\infty}$-algebras over $\mathbb{R}$, rather than as smooth objects living over $M$. But the equivalence used to prove Theorem 1.1 is local, in that the formulas are compatible with restriction maps, so we have in fact proven that the two algebras are equivalent as objects living over $M$. We anticipate that another 
natural language to convey this equivalence is to express $\mathcal{F}_{p}(M) \simeq \operatorname{Cone}\left(\omega^{p}\right)$ as an equivalence of algebroids over $M$, rather than just algebras over $\mathbb{R}$.

Notation: Later in this section, we will utilize categories and their $\infty$ categorical enhancements. We will employ a (somewhat uncommon) convention that $\underline{C}$ denotes a strict category, while $\mathrm{C}$ will denote some natural $\infty$-categorical enhancement. For instance, cdga denotes the category whose objects are commutative differential graded algebras (cdgas), and whose morphisms are maps of cdgas. The notation, cdga, without the underline, will be the $\infty$-category.

\subsection{On objects}

Here is a corollary of Theorem 1.1, which further illustrates the topological invariance of $\mathcal{F}$ and $\mathcal{C}$ :

Corollary 6.2. Let $\omega$ and $\omega^{\prime}$ be two symplectic forms on $M$, and let $\mathcal{F}_{p}(\omega)$ and $\mathcal{F}_{p}\left(\omega^{\prime}\right)$ denote the $A_{\infty}$-algebras associated to each. If $[\omega]=\left[\omega^{\prime}\right] \in$ $H^{2}(M ; \mathbb{R})$, then there is an equivalence of $A_{\infty}$-algebras $\mathcal{F}_{p}(\omega) \simeq \mathcal{F}_{p}\left(\omega^{\prime}\right)$ for every $p$.

Proof. It suffices to show an equivalence $\operatorname{Cone}\left(\omega^{p+1}\right) \simeq \operatorname{Cone}\left(\omega^{\prime p+1}\right)$. More generally, let $\zeta$ and $\zeta^{\prime}$ be two even-degree elements of a cdga that define the same cohomology class. We write an element of Cone $(\zeta)$, and its differential, as

$$
\eta \oplus \theta \xi, \quad d(\eta \oplus \theta \xi)=d \eta+\zeta \xi \oplus-\theta d \xi
$$

and likewise for $\operatorname{Cone}\left(\zeta^{\prime}\right)$ :

$$
\eta \oplus \theta^{\prime} \xi, \quad d\left(\eta \oplus \theta^{\prime} \xi\right)=d \eta+\zeta^{\prime} \xi \oplus-\theta^{\prime} d \xi
$$

Let $\lambda$ be an element of the cdga such that $d \lambda=\zeta^{\prime}-\zeta$. Then consider the map

$$
\phi: \eta \oplus \theta \xi \mapsto(\eta-\lambda \xi) \oplus \theta^{\prime} \xi
$$

This is an equivalence of cdgas - one can easily check it is both a chain map and an algebra map, and an inverse chain map is given by

$$
\eta \oplus \theta^{\prime} \xi \mapsto(\eta+\lambda \xi) \oplus \theta \xi
$$

Remark 6.3. In particular, this shows that those invariants from [17], [18], [16] expressed entirely in terms of the $A_{\infty}$-equivalence class of $\mathcal{F}_{p}$ (for example, the filtered cohomologies) are invariants only of the cohomology class 
of $\omega$, rather than on $\omega$ itself. We present an informal explanation: Usually, invariants that are sensitive to $\omega$ utilize the fact that one can detect when certain objects are positive with respect to $\omega$. (For example, one can define when an almost-complex structure $J$ defines a positive definite Riemannian metric when intertwined with $\omega$, and one can thus make statements about how spaces of positive curves (such as holomorphic curves) behave.) Since $\mathcal{F}_{0}$ by definition only sees those forms which have no $\omega$ factor, $\mathcal{F}_{0}$ is blind to such measures of positivity.

The proof of the following is straightforward:

Proposition 6.4. For all $p \geq 0$, the maps

$$
\operatorname{Cone}\left(\omega^{p+1}\right) \stackrel{q}{\rightarrow} \operatorname{Cone}\left(\omega^{p}\right), \quad q(\eta \oplus \theta \xi)=\eta \oplus \tilde{\theta} \xi \omega .
$$

are maps of cdgas; here, $\tilde{\theta}$ is the formal variable of Cone $\left(\omega^{p}\right)$ for which $d \tilde{\theta}=\omega^{p}$.

As a result, we can fill in the dotted arrow below by composing the solid arrows, which are all maps of $A_{\infty}$-algebras:

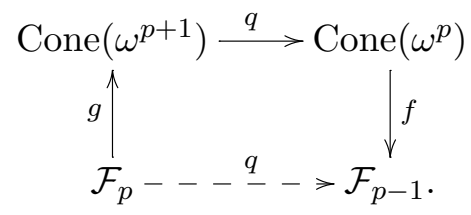

Corollary 6.5. For all $p \geq 0$, there are natural maps of $A_{\infty}$-algebras

$$
\mathcal{F}_{p} \rightarrow \mathcal{F}_{p-1}
$$

Thus to every manifold $M$ we can associate a homotopy-commutative diagram of $A_{\infty}$-algebras as follows:

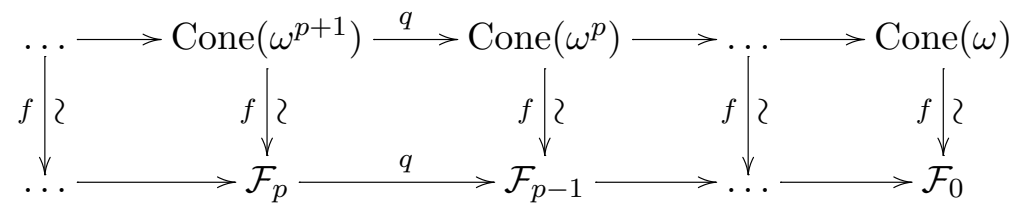

One likewise has a homotopy commutative diagram where each downward $f$ is replaced by an upward-pointing $g$. 
Remark 6.6. Recall from commutative algebra that for any primitive ideal $(f) \subset R$, one always has a sequence of commutative ring maps

$$
R /\left(f^{p+1}\right) \rightarrow R /\left(f^{p}\right)
$$

which cut out higher-degree infinitesimal neighborhoods around the locus $f=0$. The above results, particularly Proposition 6.4, are a cdga analogue. So the sequence of algebra maps (4) may be thought of as a sequence of neighborhoods around the locus where $\omega \in \Omega^{2}(M)$ vanishes. ${ }^{4}$

\subsection{On morphisms}

Note that if $f:(M, \omega) \rightarrow\left(M^{\prime}, \omega^{\prime}\right)$ is any smooth map such that $f^{*} \omega^{\prime}=\omega$, one has an induced map of cdgas

$$
f_{\text {Cone }}^{*}: \operatorname{Cone}\left(\omega^{\prime p+1}\right) \rightarrow \operatorname{Cone}\left(\omega^{p+1}\right), \quad \eta \oplus \theta^{\prime} \xi \mapsto f^{*} \eta \oplus \theta f^{*} \xi
$$

Here, $f^{*} \eta$ and $f^{*} \xi$ are the usual pullbacks of differential forms.

Now we claim that the maps of Proposition 6.4 are natural; by naturality, we mean that given any smooth map $f: M \rightarrow M^{\prime}$ such that $f^{*} \omega^{\prime}=\omega$, the diagram

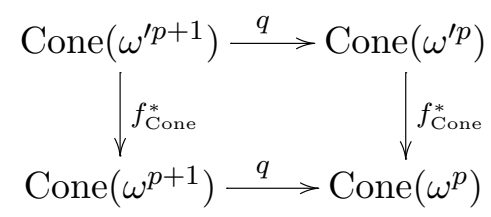

commutes on the nose. What this shows is that maps between symplectic manifolds induce maps between the sequences of algebras $\left(\operatorname{Cone}\left(\omega^{p+1}\right)\right)_{p \geq 0}$. So our work so far has lead to the following functorial description:

Let cdga be the category whose objects are cdgas, and whose morphisms are maps of cdgas. We let $\mathbb{Z}_{>0}$ denote the usual poset, considered as a category. We let $\operatorname{Fun}\left(\mathbb{Z}_{\geq 0}^{\text {op }}\right.$, cdga $)$ denote the functor category, whose morphisms are natural transformations. Finally, let Symp denote the category whose objects are symplectic manifolds, and whose morphisms are smooth maps respecting the symplectic forms.

Proposition 6.7. The assignments

- $M \mapsto\left(\ldots \rightarrow \operatorname{Cone}\left(\omega^{p+1}\right) \rightarrow \operatorname{Cone}\left(\omega^{p}\right) \rightarrow \ldots \rightarrow \operatorname{Cone}(\omega)\right)$ and

\footnotetext{
${ }^{4}$ Not as a locus on $M$, but as a locus on stacky points on the derived stack represented by Cone $(\omega)$.
} 
- $f \mapsto f_{\text {Cone }}^{*}$ from (37)

define a functor

$$
\underline{\text { Symp }} \rightarrow \operatorname{Fun}\left(\mathbb{Z}_{\geq 0}^{\text {op }}, \text { cdga }\right) .
$$

Proof. Identities are obviously sent to identities, since id ${ }^{*} \eta=\eta$ for differential forms. Composition is respected by the definition of $f_{\text {Cone }}^{*}$ in (37).

\subsection{On orthogonal homotopies}

Just as de Rham forms respect smooth homotopies, it is natural to ask whether Cone $\left(\omega^{\bullet+1}\right)$ respects certain kinds of homotopies between symplectic maps. While the assignment respects other kinds of smooth homotopies if one just remembers Cone $\left(\omega^{\bullet+1}\right)$ as a cdga, we have purposefully restricted ourselves to a class of smooth homotopies for which the homotopy formulas can be expressed in terms of local operators-i.e., for which we can stay in the $C^{\infty}$ world.

Definition 6.8. Let $F: M \times \Delta^{1} \rightarrow M^{\prime}$ be a smooth map, and let $\pi$ : $M \times \Delta^{1} \rightarrow M$ be the projection. Endow both $M$ and $M^{\prime}$ with symplectic forms. We will say that $F$ is an orthogonal homotopy of symplectic maps, or orthogonal homotopy for short, if

$$
F^{*} \omega^{\prime}=\pi^{*} \omega .
$$

Remark 6.9. The condition of being an orthogonal homotopy is a rigid one. The condition implies that

$$
F^{*} \omega^{\prime}\left(\partial_{t}, v\right)=\omega^{\prime}\left(D F\left(\partial_{t}\right), D F(v)\right)
$$

for any $v \in T M$. Hence for any time $t$, the vector $D F\left(\partial_{t}\right)$ must always be in the symplectic orthogonal to the image of $F_{t}(M)$. In particular, a homotopy obtained by pre-composing a symplectic immersion $j: M \rightarrow M^{\prime}$ by a symplectic isotopy of $M$ is not an example of an orthogonal homotopy unless the isotopy is trivial (i.e., constant).

Example 6.10. If one is given a fibration $E \rightarrow B$ where $E$ is symplectic and the symplectic form renders each fiber symplectic, then the symplectic orthogonals to each fiber determine a horizontal distribution. Parallel transport defines an orthogonal homotopy.

Remark 6.11. We are not aware if the notion of orthogonal homotopy has appeared elsewhere in the literature; we concocted it as a natural notion that induces homotopies between maps on cones (see Proposition 6.12 below). 
Proposition 6.12. If $F: M \times \Delta^{1} \rightarrow M^{\prime}$ is an orthogonal homotopy, then $F$ induces a homotopy of chain maps between $\left(F_{0}\right)_{\text {Cone }}^{*}$ and $\left(F_{1}\right)_{\text {Cone }}^{*}$.

Recall how one normally proves that a homotopy $F: M \times \Delta^{1} \rightarrow M^{\prime}$ induces a homotopy of chain maps between $F_{0}^{*}$ and $F_{1}^{*}$ on differential forms: One defines a degree -1 map

$$
H: \Omega^{\bullet}\left(M^{\prime}\right) \rightarrow \Omega^{\bullet-1}(M), \quad H\left(\eta^{\prime}\right):=\int_{0}^{1}\left(\iota_{\partial_{t}} F^{*} \eta^{\prime}\right) d t
$$

where $t$ is a coordinate for $\Delta^{1}$. Then $H$ satisfies

$$
H d+d H=F_{1}^{*}-F_{0}^{*}
$$

and hence exhibits a chain homotopy from $F_{1}^{*}$ to $F_{0}^{*}$.

So, given a smooth homotopy $F: M \times \Delta^{1} \rightarrow M^{\prime}$, define a degree -1 map as follows:

$$
\tilde{H}: \operatorname{Cone}\left(\omega^{\prime}\right)^{\bullet} \rightarrow \operatorname{Cone}(\omega)^{\bullet-1}, \quad \eta \oplus \theta^{\prime} \xi \mapsto H(\eta) \oplus-\theta H(\xi)
$$

Lemma 6.13. Let $F: M \times \Delta^{1} \rightarrow M^{\prime}$ be a smooth homotopy, and let $\tilde{H}$ be the operator defined in (40). $\tilde{H}$ exhibits a homotopy between the pullbacks $\left(F_{0}\right)_{\text {Cone }}^{*}$ and $\left(F_{1}\right)_{\text {Cone }}^{*}$ as defined in (37) if and only if one has

$$
H\left(\omega^{\prime} \xi\right)=\omega H(\xi)
$$

Proof of Lemma 6.13. We see that

$$
\begin{aligned}
\tilde{H} d+ & d \tilde{H}\left(\eta \oplus \theta^{\prime} \xi\right) \\
& =\tilde{H}\left(d \eta+\omega^{\prime} \xi \oplus-\theta^{\prime} d \xi\right)+d(H \eta \oplus-\theta H(\xi)) \\
& =(H d \eta \oplus \theta H d \xi)+H\left(\omega^{\prime} \xi\right) \oplus 0+d H \eta \oplus \theta d H(\xi)-\omega H(\xi) \oplus 0 \\
& =(H d+d H) \eta \oplus \theta(H d+d H) \xi+\left(H\left(\omega^{\prime} \xi\right)-\omega H(\xi)\right) \oplus 0 \\
& =\left(F_{1}^{*}-F_{0}^{*}\right) \eta \oplus \theta\left(F_{1}^{*}-F_{0}^{*}\right) \xi+\left(H\left(\omega^{\prime} \xi\right)-\omega H(\xi)\right) \oplus 0 \\
& =\left(\left(F_{1}\right)_{\text {Cone }}^{*}-\left(F_{0}\right)_{\text {Cone }}^{*}\right)(\eta \oplus \theta \xi)+\left(H\left(\omega^{\prime} \xi\right)-\omega H(\xi)\right) \oplus 0 .
\end{aligned}
$$

Proof of Proposition 6.12. Writing out (39), the lefthand side of (41) becomes

$$
H\left(\omega^{\prime} \xi\right)=\int_{0}^{1} \iota_{\partial_{t}} F^{*}\left(\omega^{\prime} \xi\right) d t
$$




$$
\begin{aligned}
& =\int_{0}^{1}\left(\iota_{\partial_{t}} F^{*} \omega^{\prime}\right) \wedge F^{*} \xi+F^{*} \omega^{\prime} \wedge \iota_{\partial_{t}} F^{*} \xi d t \\
& =\int_{0}^{1}\left(\iota_{\partial_{t}} F^{*} \omega^{\prime}\right) \wedge F^{*} \xi+F^{*} \omega^{\prime} \wedge \iota_{\partial_{t}} F^{*} \xi d t
\end{aligned}
$$

while the righthand side of (41) is given by

$$
\omega H(\xi)=\omega \int_{0}^{1} \iota_{\partial_{t}}\left(F^{*} \xi\right) d t .
$$

Now we show that a sufficient condition for (41) to hold is the given by (38).

The condition (38) implies that for every time $t, F_{t}^{*} \omega^{\prime}=\omega$. This is because, letting $i_{t}: M \hookrightarrow M \times \Delta^{1}$ be the inclusion at time $t$, we have that

$$
\left(F \circ i_{t}\right)^{*} \omega^{\prime}=i_{t}^{*} F^{*} \omega^{\prime}=i_{t}^{*} \pi^{*} \omega=\left(\pi \circ i_{t}\right)^{*} \omega=\mathrm{id}_{M}^{*} \omega=\omega .
$$

In other words, $F$ is a homotopy through maps that respect the symplectic form. Even more strongly, the definition implies that for all $v \in T M$, we have

$$
\left(\iota_{\partial_{t}} F^{*} \omega^{\prime}\right) v=F^{*} \omega^{\prime}\left(\partial_{t}, v\right)=\pi^{*} \omega\left(\partial_{t}, v\right)=\omega\left(D \pi\left(\partial_{t}\right), D \pi(v)\right)=\omega(0, D \pi(v))=0 .
$$

Hence the definition implies that

$$
\iota_{\partial_{t}} F^{*} \omega^{\prime}=0
$$

Because $F_{t}^{*} \omega^{\prime}=\omega$ for all $t$, this is enough for (41) to hold.

Remark 6.14. One would like to further say that orthogonal homotopies determine homotopies between the algebra maps, rather than just the chain maps. However, we have not delved into the algebraic theory for homotopies in the smooth category for the reasons mentioned in Remark 6.1; for instance, the usual definition for a homotopy between cdga maps uses polynomial forms on the 1-simplex, which are only suitable when one deals with smooth homotopies whose dependence on $t$ happens to be polynomial. (I.e., not very often.)

\subsection{Sheaf property}

Note that being primitive is a local property. Moreover, $\partial_{-}$and $\partial_{+}$are local operators, so the differentials of $\mathcal{F}$ are compatible with the restriction of 
differential forms from one open subset to another. All this data obviously forms a sheaf of cochain complexes; we prove that they also form a sheaf which is robust with respect to the homotopy theory of $A_{\infty}$-algebras:

Theorem 6.15. The assignment $U \mapsto \mathcal{F}(U)$ is a sheaf on $M$ with values in the $\infty$-category of $A_{\infty}$-algebras.

We recall that if $\mathcal{C}$ is an $\infty$-category with limits, a presheaf on $M$ with values in $\mathcal{C}$ is a functor

$$
\mathcal{F}: \text { Open }(M)^{\text {op }} \rightarrow \mathcal{C}
$$

Here, Open $(M)$ is the nerve of the category of open subsets of $M$ (i.e., the $\infty$-category associated to the usual 1-category Open $(M)$ of open subsets). We call $\mathcal{F}$ a sheaf if the following holds: For any open cover $\left\{U_{\alpha}\right\}$ of any open set $U$, the associated augmented cosimplicial diagram

$$
\mathcal{F}(U) \longrightarrow \prod_{\alpha} \mathcal{F}\left(U_{\alpha}\right) \Longrightarrow \prod_{\alpha, \beta} \mathcal{F}\left(U_{\alpha} \cap U_{\beta}\right) \Longrightarrow \ldots
$$

is a limit diagram in $\mathcal{C}$.

Remark 6.16. Since $\mathcal{C}$ is an $\infty$-category, when we say "limit" in $\mathcal{C}$, there is only one notion of limit - if $\mathcal{C}$ arises from a model category, for instance, a limit in $\mathcal{C}$ is equivalent to the appropriate homotopy limit computed in the model category.

Proof. Let $\mathrm{A}_{\infty} \mathrm{Alg}$ be the $\infty$-category of $A_{\infty}$-algebras over $\mathbb{R}$. Then the assignment $U \mapsto \mathcal{F}(U)$ defines a functor

$$
\mathcal{F}: \text { Open }(M)^{\text {op }} \rightarrow \mathrm{A}_{\infty} \mathrm{Alg}
$$

(In fact, it factors through the strict 1-category $A_{\infty} A l g$. )

Since the forgetful functor $A_{\infty} A l g \rightarrow$ Chain creates limits, one need only prove that this augmented diagram is a limit diagram in the $\infty$-category of cochain complexes. But in general, if $\mathcal{F}$ is a sheaf of cochain complexes in the strict sense (meaning that the augmented diagram is a limit diagram in the 1-category $\underline{\text { Chain) }}$ in which the degree $n$ presheaf $\mathcal{F}^{n}$ is a soft sheaf for every $n$, then $\mathcal{F}$ is itself a homotopy sheaf [11]. That is, the augmented diagram in cochain complexes is a limit diagram in the $\infty$-category Chain.

So one simply needs to check that $\mathcal{F}_{p}$ is a complex of soft sheaves. This is elementary - each $\mathcal{F}_{p}^{k}$ is soft because differential forms on a closed subset can extend to a differential form globally. $\mathcal{F}_{p}$ is a sheaf for the same reasons that differential forms form a sheaf. 
Remark 6.17. The equivalences $\mathcal{F} \rightarrow \mathcal{C}$ are natural for open inclusions by Theorem 1.1; so one could also prove the (homotopy) sheaf property by showing that $U \mapsto \mathcal{C}(U)$ is a homotopy sheaf. This can be proved using the exact same argument as above.

Remark 6.18. In fact, if one considers the $\infty$-category

$$
\operatorname{Fun}\left(\mathbb{Z}_{\geq 0}^{\text {op }}, A_{\infty} A l g\right)
$$

then we see that $\mathcal{F}$ defines a sheaf on $M$ with this target $\infty$-category-this is because limits in a diagram category are computed pointwise (i.e., one need only check that for every $n \in \mathbb{Z}_{\geq 0}$, the resulting cosimplicial diagram is a limit diagram). Note that $A_{\infty} A l g$ contrasts with cdga, which did not incorporate higher homotopies. And if one uses the model of filtered forms using the cone construction - utilizing the equivalence of Theorem 1.1-then one can make a stronger statement: The assignment

$$
U \mapsto\left(\ldots \rightarrow \operatorname{Cone}\left(\omega^{p+1}\right)(U) \rightarrow \operatorname{Cone}\left(\omega^{p}\right)(U) \rightarrow \ldots \rightarrow \operatorname{Cone}(\omega)(U)\right)
$$

is a homotopy sheaf in the $\infty$-category $\operatorname{Fun}\left(\mathbb{Z}_{\geq 0}^{\mathrm{op}}\right.$, cdga $)$ where we remove the underline.

\subsection{As sheaf cohomology}

Recall Leray's theory of sheaf cohomology, which in Grothendieck's language is obtained as a right derived functor of the global sections functor $F \mapsto F(M)$. It is a consequence of the de Rham-Weil theorem that these cohomology groups can be computed by taking an acyclic (rather than injective) resolution.

One sees immediately that the de Rham forms $\Omega^{k}(M)$ are soft, as we mentioned in the proof of Theorem 6.15. Likewise, it is clear that each sheaf $\mathcal{F}_{p}^{k}$ or Cone $\left(\omega^{p+1}\right)^{k}$ is a soft sheaf on $M$. So we must ask - is there a natural sheaf for which these complexes are a resolution?

Though the following discussion is valid using the $A_{\infty}$-algebra $\mathcal{F}$ as well, we will focus on the sheaf Cone $\left(\omega^{p+1}\right)$. The following should be compared to Proposition 3.3 of [16]:

Proposition 6.19. Locally on $M$, the sequence

$$
\Omega^{k-1} \oplus \theta \Omega^{k-2 p-2} \rightarrow \Omega^{k} \oplus \theta \Omega^{k-2 p-1} \rightarrow \Omega^{k+1} \oplus \theta \Omega^{k-2 p}
$$

is exact for $k \geq 2 p+2$. 
Proof. Evaluate the sequence on some open set $U$ which is diffeomorphic to $\mathbb{R}^{2 n}$. If $\eta \oplus \theta \xi$ is in the kernel of $\left.d\right|_{U}$, then we know $d \eta=-\omega^{p+1} \xi$ and $d \xi=0$. The Poincaré Lemma says we can find $\xi^{\prime}$ such that $-d \xi^{\prime}=\xi$. Moreover, $\eta-\omega^{p+1} \xi^{\prime}$ is closed, so the Poincaré Lemma also allows us to find $\eta^{\prime}$ so that $d \eta^{\prime}=\eta-\omega^{p+1} \xi^{\prime}$. Thus $\eta \oplus \theta \xi=d\left(\eta^{\prime} \oplus \theta \xi^{\prime}\right)$.

The exactness fails at the $2 p+1$ group of Cone $\left(\omega^{p+1}\right)$. Of course, the complex is exact at degrees $1 \leq k \leq 2 p$ by the usual Poincaré Lemma. For this reason, the sheaf cohomology description is cleanest when $p=0$, though one can easily formulate the analogue for higher $p$.

Definition 6.20. For any open set $U \subset M$, let $\operatorname{Prim}^{\prime}(U)$ be the vector space whose elements are pairs $(\eta, b)$ where $b$ is a locally constant function on $U$, and $\eta$ is a 1 -form such that $d \eta=-\left.b \omega\right|_{U}$. We let Prim denote the sheafification of Prim'.

Proposition 6.21. Cone $(\omega)$ is a soft resolution of the two-term complex of sheaves

$$
\Omega^{0} \rightarrow \text { Prim, } \quad g \mapsto(d g, 0) .
$$

Proof. We simply need to show that the inclusion

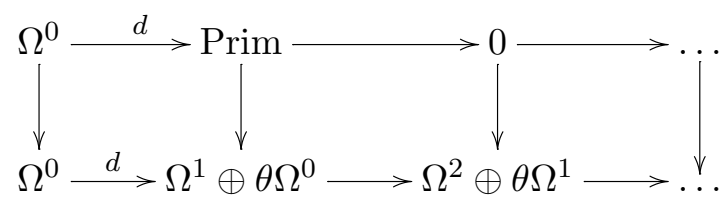

is an equivalence of complexes of sheaves - that is, that the map induces an isomorphism of cohomology sheaves $\mathcal{H}^{k}$. We already saw in Proposition 6.19 that the Cone $(\omega)$ complex is indeed locally acyclic at $k \geq 2$. So one need only show an isomorphism at $\mathcal{H}^{0}$ and $\mathcal{H}^{1}$. The isomorphism at $\mathcal{H}^{0}$ is obvious, as the inclusion naturally identifies the kernels of $d$ - the locally constant functions. The isomorphism at $\mathcal{H}^{1}$ is also obvious, as Prim' ${ }^{\prime}$ is defined to locally be the kernel of the differential $\Omega^{1} \oplus \theta \Omega^{0} \rightarrow \Omega^{2} \oplus \theta \Omega^{1}$.

Remark 6.22. A similar proof shows the trivial result that Cone $\left(\omega^{p+1}\right)$ is a soft replacement for the complex of sheaves

$$
\Omega^{0} \rightarrow \ldots \rightarrow \Omega^{2 p} \rightarrow \operatorname{ker}\left(\left.d\right|_{\Omega^{2 p+1} \oplus \theta \Omega^{0}}\right) \rightarrow 0
$$

which, of course, is soft away from the last non-zero entry. 


\subsection{Looking forward: Weinstein functoriality}

Each sequence of cdgas Cone $\left(\omega^{\bullet+1}\right)$ depends only on the cohomology class of $\omega$. However, the equivalence $\operatorname{Cone}(\omega) \simeq \operatorname{Cone}\left(\omega^{\prime}\right)$ from Corollary 6.2 relied on a choice of $\lambda$ such that $d \lambda=\omega^{\prime}-\omega$. At this point, we see two doorways into further structure:

1. The first appears when we recall that maps which respect $\omega$ are only one kind of morphism between symplectic manifolds. As advocated by Weinstein, a more natural notion of morphism is given by submanifolds of $M \times M^{\prime}$. In general, graphs of symplectomorphisms give rise to Lagrangian submanifolds of $M \times M^{\prime}$, but the fact that Cone $\left(\omega^{\bullet+1}\right)$ is functorial with respect to all maps respecting $\omega$ suggests the following: isotropic submanifolds of $M \times M^{\prime}$ should also be included in the class of morphisms we consider. (Graphs of smooth maps respecting $\omega$ are, in general, isotropic.) In other words, we should look for a Weinstein functoriality with respect to isotropic correspondences between symplectic manifolds - not just smooth maps respecting $\omega$.

2. The second is that specifying forms $\lambda$ that realize the equality $[\omega]=\left[\omega^{\prime}\right]$ is a natural piece of data to include in whatever kind of functoriality we consider. This is a picture one has seen before: For instance, in the framework of [15], derived symplectic manifolds and their Lagrangians all carry with them additional data showing how symplectic forms are closed, and how their restrictions to Lagrangians are null.

Following through on the second doorway goes beyond the scope of our current paper, as we would need to seriously develop the machinery for derived smooth manifolds, for their cotangent complexes, and for writing down a stack classifying closed 2-forms. (Roughly, we would need a $C^{\infty}$ analogue of all the basic ingredients set up in [15] in the context of derived algebraic geometry.) So here we merely open the first door.

Remark 6.23 (Motivation for Weinstein functoriality). It is an idea going back at least to Weinstein [20] that, rather than just symplectomorphisms, Lagrangian correspondences $L \subset M_{1} \times M_{2}$ ought to behave like morphisms in the category of symplectic manifolds. This idea is compatible with the notion that boundary conditions for field theories define Lagrangian submanifolds in the space of classical solutions (see, for example, the AtiyahFloer conjecture), and many efforts are being made to formalize this idea for Fukaya categories, where one expects Lagrangian correspondences to define a bimodule between the Fukaya categories of $M_{1}$ and $M_{2}$. 
Let $j: L \rightarrow M_{1} \times M_{2}$ be an immersion. Also assume that $j^{*} \omega_{1}=j^{*} \omega_{2}$, so that $L$ is an immersed isotropic submanifold of $\left(M_{1} \times M_{2},-\omega_{1} \oplus \omega_{2}\right)$.

Definition 6.24. We let $\operatorname{Cone}_{L}\left(\omega^{p}\right)$ denote the mapping cone of the map

$$
\Omega(L)[-2 p] \stackrel{j^{*} \omega_{2}}{\longrightarrow} \Omega(L) .
$$

Remark 6.25. One should expect a construction of $\mathrm{Cone}_{L}$ having the same flavor as the construction of the $\mathcal{F}_{p}$. We do not know of any such construction at present.

Because $j$ is an isotropic immersion, we see immediately:

Proposition 6.26. Let Cone $_{M_{i}}\left(\omega_{i}^{p+1}\right)$ denote the cone algebra for $M_{i}$. The composite smooth maps $L \rightarrow M_{1} \times M_{2} \rightarrow M_{i}$ induce maps $\Omega\left(M_{i}\right) \rightarrow \Omega(L)$, and these in turn induce maps of cdgas

$$
\operatorname{Cone}_{M_{1}}\left(\omega_{1}^{p+1}\right) \rightarrow \operatorname{Cone}_{L}\left(\omega^{p+1}\right) \leftarrow \operatorname{Cone}_{M_{2}}\left(\omega_{2}^{p+1}\right) .
$$

In other words, Cone $_{L}\left(\omega^{p+1}\right)$ is a bimodule for each cone algebra.

In particular, any Lagrangian immersion defines a bimodule between the algebras associated to each $M_{i}$. Note also that if $j: L \rightarrow M_{1} \times M_{2}$ is not strictly an isotropic map, but if one can specify a form $\lambda_{L}$ such that $d \lambda_{L}=j^{*} \omega_{2}-j^{*} \omega_{1}$, then this specifies an equivalence

$$
\operatorname{Cone}_{L}\left(\omega_{1}\right) \simeq \operatorname{Cone}_{L}\left(\omega_{2}\right)
$$

hence one still has a bimodule over the two cone algebras given by the $M_{i}$. This is exactly the kind of data that we should witness when following through with door (2), which we leave for later work.

Finally, we also leave for later work the proof of Weinstein functoriality in (1), which likewise requires a development of some derived smooth geometry to incorporate non-transverse intersections of Lagrangian and isotropic correspondences. The expectation, of course, is that compositions of Lagrangian correspondences are taken to tensor products of bimodules under the functor $\mathcal{F}_{\bullet}$ (or equivalently, the functor $\operatorname{Cone}\left(\omega^{\bullet+1}\right)$ ).

\section{Appendix A. Proof that Cone $(\omega)$ is equivalent to differential forms on a sphere bundle}

Assume $[\omega]$ is an integral cohomology class on $M$. Then $[\omega]$ classifies a complex line bundle $L$ on $M$, and hence a circle bundle. More generally, the 
higher powers $\left[\omega^{p+1}\right]$ are the top Chern classes of the vector bundles $L^{\oplus p+1}$. There is a natural smooth sphere bundle $E_{p}$ associated to this vector bundle.

In this appendix, we give a proof of the following:

Theorem A.1. Let $M$ be an arbitrary symplectic manifold, and $\omega$ a symplectic form defining an integral cohomology class. Then there is an equivalence of cdgas

$$
\Omega\left(E_{p}\right) \simeq \operatorname{Cone}\left(\omega^{p+1}\right) .
$$

Remark A.2. This result is obvious if $M$ is simply-connected-by rational homotopy theory, the cone is a standard cdga model for forms on the total space of an odd-dimensional sphere bundle.

Remark A.3. Fix a real number $k \neq 0$. Note that if one scales $\omega$ to $k \omega$, one has an isomorphism of cdgas as follows: (replaced $\zeta$ by $\xi$ )

$$
K: \operatorname{Cone}\left(\omega^{p+1}\right) \simeq \operatorname{Cone}\left(k^{p+1} \omega^{p+1}\right), \quad \eta \oplus \theta \xi \mapsto \eta \oplus \frac{1}{k^{p+1}} \theta \xi .
$$

The proof of the theorem uses the following easy lemma:

Lemma A.4. Theorem A.1 holds when $M$ is contractible.

Proof of Lemma. When $M$ is contractible, $E_{p}$ is trivial, and homotopy equivalent to $S^{2 p+1}$. Hence we can choose a global $2 p+1$-form called $\underline{\theta}$ that realizes a generator for the cohomology of the fiber (and hence of $E_{p}$ ). Then we claim that the cdga map

$$
\text { Cone }\left(\omega^{p+1}\right)=\Omega^{\bullet}(M) \oplus \theta \Omega^{\bullet-2 p-1}(M) \rightarrow \Omega\left(E_{p}\right), \quad \eta \oplus \theta \xi \mapsto \pi^{*} \eta+\underline{\theta} \wedge \pi^{*} \xi
$$

exhibits the equivalence. Note it is obviously a surjection on cohomology, as the generators of $H^{0}\left(S^{2 p+1}\right)$ and $H^{2 p+1}\left(S^{2 p+1}\right)$ are hit by

$$
1 \oplus 0 \quad \text { and } \quad 0 \oplus \theta 1,
$$

respectively. (Here, "1" is the constant 0 -form with value 1.) It is further an injection on cohomology, because the long exact sequence in cohomology for a cone (along with the contractibility of $M$ ) shows that these are the only possible cohomology generators in Cone $(\omega)$.

Proof of Theorem A.1. $E_{p}$ is an oriented sphere bundle, being the sphere bundle associated to a complex vector bundle. We let $\underline{\theta} \in \Omega^{2 p+1}\left(E_{p}\right)$ denote the global angular form on $E_{p}$. (For the existence and properties of the global angular form of a sphere bundle, see Section 11 of Bott-Tu [1].) Being 
a global angular form, $\underline{\theta}$ has the following two properties: (i) $\underline{\theta}$ restricts to a generator of $H^{2 p+1}\left(S^{2 p+1}\right)$ on each fiber of $E_{p}$, and (ii) $d \underline{\theta}=\pi^{*}\left(\omega^{p+1}\right)$. Note property (ii) follows because $\omega^{p+1}$ is a representative of the Euler class of $E_{p}$.

So the same map as in (42) is a map of cdgas.

Let $\mathcal{V}=\left\{V_{\alpha}\right\}$ be a good cover of $M$. If $\pi: E_{p} \rightarrow M$ is the projection map of the fibration, the open sets $U_{\alpha}=\pi^{-1}\left(V_{\alpha}\right)$ form an open cover of $E_{p}$, where each non-empty intersection $U_{\alpha_{0}, \ldots, \alpha_{r}}:=U_{\alpha_{0}} \cap \ldots \cap U_{\alpha_{r}}$ is homotopy equivalent to the fiber sphere. Consider the Cech-de Rham double complex, which is the $E_{0}$ page of the spectral sequence given by the usual filtration on on $\Omega\left(E_{p}\right)$.

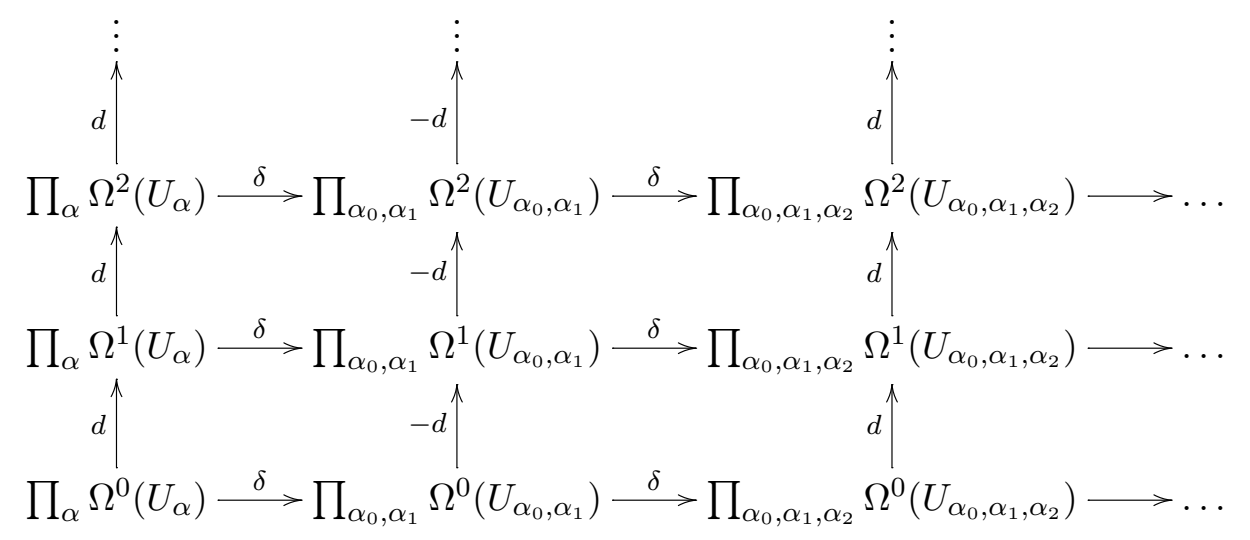

The advantage of working in the smooth world (and hence using the de Rham model) is that this double complex has a total complex which is a cdga - after all, this is the Cech complex of a (pre)sheaf of cdgas. ${ }^{5}$ Moreover, the usual augmentation from $\Omega^{\bullet}\left(E_{p}\right)$ gives a map of cdgas from $\Omega\left(E_{p}\right)$ to the total complex, and this is an equivalence of cdgas. (For instance, each row of this $E_{0}$ page is exact by the existence of partitions of unity. This is one classical way to see that the Čech-de Rham double complex computes the de Rham cohomology algebra of the total space.)

Now we note that the map of cdgas (42) is local-it is compatible with restriction maps. Hence (42) induces a map of double complexes. For the sake of having a name, we will call the domain double complex the " $V$ double complex." At the $r$ th column and sth row, the map from the $V$ double complex to the Cech-de Rham complex is given by

$$
\Omega^{s}\left(V_{\alpha_{0}, \ldots, \alpha_{r}}\right) \oplus \theta \Omega^{s-2 p-1}\left(V_{\alpha_{0}, \ldots, \alpha_{r}}\right) \rightarrow \Omega^{s}\left(U_{\alpha_{0}, \ldots, \alpha_{r}}\right), \quad \eta \oplus \theta \xi \mapsto \pi^{*} \eta+\underline{\theta} \wedge \pi^{*} \xi .
$$

\footnotetext{
${ }^{5}$ As opposed to having to keep track of $E_{\infty}$ structures on singular cochains.
} 
But this is the map used in the Lemma, hence an equivalence of cdgas. Thus the vertical differentials $d$ of the $V$ double complex compute the same $E_{1}$ page of the spectral sequence associated to the Cech-de Rham double complex. Importantly, the horizontal differentials of the $E_{2}$ page are also equal - this is where one uses the existence of the global angular form. Specifically, the horizontal Čech complexes have compatible differentials precisely because a single global form restricts to the generators along each open set.

The last (and only other) page at which one has a differential is the $E_{2 p+2}$ page, where the differential is precisely given by the map called "wedge with $\omega^{p+1}$." (This is by definition of the differential of Cone $\left(\omega^{p+1}\right)$, which leads to this differential in the $V$ double complex, and by a well-known property of the Euler form for the Cech-de Rham double complex.)

This completes the proof.

\section{References}

[1] R. Bott and L. Tu, Differential forms in algebraic topology. SpringerVerlag (1982). MR658304

[2] F. Bourgeois, A Morse-Bott approach to contact homology. Stanford Univ. Ph.D. Thesis, ProQuest LLC (2002). MR2703292,

[3] C. Brav and T. Dyckerhoff, Relative Calabi-Yau structures. (2016), arXiv:1606.00619.

[4] K. Costello, Topological conformal field theories and Calabi-Yau categories. Adv. Math. 210 (2007), 165-214. MR2298823

[5] S. K. Donaldson, Symplectic submanifolds and almost-complex geometry. J. Differential Geom. 44 (1996), 666-705. MR1438190

[6] H. Federer, Geometric measure theory. Springer-Verlag (1969). MR0257325

[7] S. Ganatra, T. Perutz, and N. Sheridan, Mirror symmetry: from categories to curve counts. (2015), arXiv:1510.03839v2.

[8] V. Ginzburg, Calabi-Yau algebras. (2006), arXiv:math.AG/0612139.

[9] P. Griffiths and J. Harris, Principles of algebraic geometry. John Wiley \& Sons, Inc. (1994). MR1288523

[10] H. Kajiura, Hiroshige, Noncommutative homotopy algebras associated with open strings. Rev. Math. Phys., 19 (2007), 1-99. MR2293085

[11] M. Kashiwara and P. Schapira, Sheaves on manifolds. Springer-Verlag (1990). MR1074006 
[12] B. Keller, Introduction to A-infinity algebras and modules. Homology Homotopy Appl. 3 (2001), 1-35. MR1854636

[13] M. Kontsevich and Y. Soibelman, Notes on $A_{\infty}$-algebras, $A_{\infty}$-categories and non-commutative geometry. In Homological mirror symmetry. Lecture Notes in Phys. 757 153-219, Springer (2009). MR2596638

[14] S.-C. Lau, L.-S. Tseng, and S.-T. Yau, Non-Kähler SYZ mirror symmetry Comm. Math. Phys. 340, (2015), 145-170. MR3395150

[15] T. Pantev, B. Toën, M. Vaquié and G. Vezzosi, Shifted symplectic structures. Publ. Math. Inst. Hautes Études Sci. 117 (2013), 271-328. MR3090262

[16] C.-J. Tsai, L.-S. Tseng and S.-T. Yau, Cohomology and Hodge theory on symplectic manifolds: III. J. Differential Geom. 103 (2016), 83-143. MR3488131

[17] L.-S. Tseng and S.-T. Yau, Cohomology and Hodge theory on symplectic manifolds: I. J. Differential Geom. 91 (2012), 383-416. MR2981843

[18] L.-S. Tseng and S.-T. Yau, Cohomology and Hodge theory on symplectic manifolds: II. J. Differential Geom. 91 (2012), 417-443. MR2981844

[19] L.-S. Tseng and S.-T. Yau, Generalized cohomologies and supersymmetry. Comm. Math. Phys. 326 (2014), 875-885. MR3173409

[20] A. Weinstein, The symplectic "category." In Differential geometric methods in mathematical physics (Clausthal, 1980). Lecture Notes in Mathematics, 905 153-219, Springer (1982). MR657441

Hiro Lee Tanaka

DePARTMENT OF MATHEMATiCs

HARVARD UNIVERSITY

CAMBridge, MA 02138

USA

E-mail address: hirolee@math.harvard.edu

Li-Sheng TSEnG

Department of Mathematics

UNIVERSITY OF CALIFORNIA

IRVINE, CA 92697

USA

E-mail address: lstseng@math.uci.edu

ReCeived November 30, 2017 